TRANSACTIONS OF THE

AMERICAN MATHEMATICAL SOCIETY

Volume 352, Number 7 , Pages 3381-3410

S 0002-9947(00)02476-4

Article electronically published on March 27, 2000

\title{
HEREDITARY CROSSED PRODUCTS
}

\author{
JEREMY HAEFNER AND GERALD JANUSZ
}

\begin{abstract}
We characterize when a crossed product order over a maximal order in a central simple algebra by a finite group is hereditary. We need only concentrate on the cases when the group acts as inner automorphisms and when the group acts as outer automorphisms. When the group acts as inner automorphisms, the classical group algebra result holds for crossed products as well; that is, the crossed product is hereditary if and only if the order of the group is a unit in the ring. When the group is acting as outer automorphisms, every crossed product order is hereditary, regardless of whether the order of the group is a unit in the ring.
\end{abstract}

\section{INTRODUCTION AND PRELIMINARIES}

Let $R$ be a Dedekind domain with quotient field $K$. Let $A$ be a finite dimensional, separable $K$-algebra and let $\Lambda$ denote an $R$-order in $A$. By a $\Lambda$-lattice, we mean a finitely generated (left) $\Lambda$-module $M$ such that $M$ is $R$-torsionfree. Let $G$ be a (finite) group and let $\Lambda$ be an $R$-order. We say that $\Lambda$ is strongly $G$-graded if $\Lambda=\bigoplus_{g \in G} \Lambda_{g}$, where each $\Lambda_{g}$ is a $\Lambda_{1}$-module and the multiplication is given by $\Lambda_{x} \cdot \Lambda_{y}=\Lambda_{x y}$ for all $x, y \in G$. A special type of strongly graded order is the crossed product. We say that $\Lambda$ is a crossed product of an order $\Delta$ by a finite group $G$ provided that $\Lambda$ is strongly $G$-graded such that $\Lambda_{1}=\Delta$ and every component $\Lambda_{g}$ contains a unit of $\Lambda$. This is equivalent to the condition that $\Lambda$ is a free $\Delta$-bimodule with basis $\{\bar{g} \mid g \in G\}$, a map $\alpha: G \rightarrow \operatorname{Aut}(\Delta)$ and a factor set $\tau \in Z^{2}\left(G, \Delta^{\bullet}\right)\left(\Delta^{\bullet}\right.$ denotes the units of $\Delta$ ) such that

$$
\bar{g} \cdot \delta=\alpha(g)(\delta) \cdot \bar{g} \text { and } \bar{g} \cdot \bar{h}=\tau(g, h) \cdot \overline{g h}
$$

for all $g, h \in G$ and $\delta \in \Delta$. We call the map $\alpha$ the action of $G$ and the map $\tau \in Z^{2}\left(G, \Delta^{\bullet}\right)$ a factor set or, more loosely, a twisting of $G$. The most general twisting of a group action would permit factor sets with values in $\Delta^{\bullet}$. We will not consider the most general situation, but will restrict our attention to factor sets with values in the units of $R$. We denote such an order by $\Delta *_{\tau}^{\alpha} G$, or by $\Delta * G$ when the maps $\alpha$ and $\tau$ are clear from context. Group algebras, twisted group algebras and skew group algebras are special cases of crossed product orders. See [12] for more details about crossed products.

The purpose of this paper is to investigate when a crossed product order is hereditary. Specifically, the hereditary problem for crossed products is to

Received by the editors February 23, 1998.

1991 Mathematics Subject Classification. Primary 16G30, 16H05, 16S35, 16W20, 16W50.

Key words and phrases. Order, finite representation type, hereditary crossed products, automorphisms.

The first author was partially supported by a grant from the National Security Agency. 
determine necessary and sufficient conditions on the group $G$, the action $\alpha$ and the twisting $\tau$ so the crossed product $\Gamma *_{\tau}^{\alpha} G$ is hereditary. We solve this hereditary problem in the global situation; specifically, we prove:

Theorem A (Haefner-Janusz, 98). Let $R$ denote a Dedekind domain whose quotient field $K$ is a global field. Let $\Gamma$ be an $R$-order inside a central simple $K$-algebra $A, \alpha: G \rightarrow \operatorname{Aut}_{R}(\Gamma)$ a group homomorphism and $\tau \in Z^{2}\left(G, R^{\bullet}\right)$. Set $\Lambda=\Gamma *_{\tau}^{\alpha} G$. Then $\Lambda$ is hereditary if and only if, for each maximal ideal $P$ that contains a prime integer $p$ dividing $|G|$, any $p$-Sylow subgroup of $G$ acts as central outer automorphisms of $\hat{\Gamma}_{P}$ (i.e., the P-adic completion of $\Gamma$ ).

Our solution relies on the fact that the investigation can be divided into two cases: the case where $G$ is acting entirely as inner automorphisms of $\Gamma$ and the case where $G$ is acting entirely as outer automorphisms of $\Gamma$. When $\Lambda=R G$, the ordinary group algebra, then $\Lambda$ is hereditary if and only if the order of $G$ is a unit in $R$. See [14, Theorem 41.1] for details. Our analysis of the inner case reveals that this result extends to crossed products. We prove:

Theorem B. Let $\Gamma \neq R$ be a maximal $R$-order in a central simple $K$ algebra, $G$ a group of order $n$, and $\alpha: G \rightarrow \operatorname{Inn}(\Delta)$ a homomorphism. For any factor set $\tau$ defined on $G$ with values in $R^{\bullet}$, the following statements are equivalent:

1. $\Gamma *_{\tau}^{\alpha} G$ is maximal.

2. $\Gamma *_{\tau}^{\alpha} G$ is hereditary.

3. $n=|G|$ is a unit of $R$.

See Theorem [2.4] This theorem, together with Example 5.14, corrects an error in the only if statement from [8, Theorem 4.2].

In contrast, the outer case is considerably different because every crossed product $\Delta * G$ is hereditary, whenever $G$ is acting as central outer automorphisms. We say that $G$ acts as central outer automorphisms provided there exists a map $\alpha: G \rightarrow$ $\operatorname{Aut}_{R}(\Delta)$ such that $\alpha(g)$ is inner if and only if $g$ is the identity; see Definition 4.1 for details. Our main result is:

Theorem C. Let $R$ be a complete local Dedekind domain with quotient field $K$ which is a local field. Let $\Delta$ be a maximal $R$-order inside a central $K$-division ring $D$. If $G$ is a group that acts on $\Delta$ as centralizing outer automorphisms via the action $\alpha: G \rightarrow \operatorname{Aut}(\Delta)$ and twisting $\tau \in$ $Z^{2}\left(G, R^{\bullet}\right)$, then the crossed product order $\Delta *_{\tau}^{\alpha} G$ is hereditary.

See Theorem 5.15 for the proof. Our proof technique is to use splitting fields to reduce to the situation where the coefficient ring $\Delta$ is a hereditary submatrix ring with entries from a commutative Dedekind domain (Theorem [3.4). In the case where $G$ acts as outer automorphisms of $\Delta$, we are able to further reduce to the case where $\Delta$ is itself commutative. In particular, we obtain the following additional result:

Theorem D. Let $\Lambda$ denote a basic, hereditary $S$-order inside $M_{n}(S)$, where $S$ is a complete, local Dedekind domain. If $G$ acts on $\Lambda$ as central outer automorphisms via the group action $\alpha: G \rightarrow \operatorname{Aut}(\Lambda)$ and factor set $\tau \in H^{2}\left(G, S^{\bullet}\right)$, then $\Lambda{ }_{\tau}^{\alpha} G$ is hereditary and has finite representation type.

See Theorem 5.13 for details. 
We close our introductory remarks by mentioning that the motivation for this hereditary problem arises from the study of when a strongly graded order $\Lambda$ has finite representation type. The order $\Lambda$ has finite representation type (FRT) provided $\Lambda$ has only finitely many non-isomorphic, indecomposable $\Lambda$-lattices. The FRT problem is to determine when a strongly $G$-graded order $\Lambda$, whose 1-component is a maximal $R$-order, has finite representation type in terms of the group $G$ and the grading. In [8], we reduced the study of strongly graded orders that have FRT to the crossed product case; that is, it suffices to determine necessary and sufficient conditions for when crossed product orders of the form $\Delta * G$, where $\Delta$ is a maximal $R$-order in a division ring, $G$ is a $p$-group, and $R$ is a complete DVR in a quotient field $K$, have finite representation type. As a result of Theorem B, we now know that whenever $G$ acts as central outer automorphisms, then $\Delta * G$ is hereditary and so has FRT.

This paper is organized as follows. In section 2, we define and use separable functors to solve the inner case and prove Theorem B. We establish a relationship in section 3 between $\Delta * G$ and $(S \otimes \Delta) * G$, where $S$ is the integral closure of $R$ inside an unramified splitting field $E$ for the division ring $D$. This allows us to pass the hereditary problem from $\Delta * G$ to a crossed product over a submatrix order inside $M_{n}(S)$. In section 4 we analyze the central outer automorphisms of $\Delta$ and $S \otimes \Delta$, and we prove Theorems $\mathrm{C}$ and D in section 5. Finally, in section 6 , we investigate the central simple case and prove the global result, Theorem A.

We consider only Dedekind domains that satisfy the Jordan-Zassenhaus Theorem (44, Theorem 24.1]); this means that the quotient field of the domain is a local or global field. All groups are finite. As in the statement of the hereditary problem, the action map of a crossed product will always be a group homomorphism and the twisting map will always be a cocycle (i.e., $\tau \in Z^{2}\left(G, R^{\bullet}\right)$ ).

\section{Separable functors and the inner CASE}

Central to all our proofs in this paper is the notion of a separable functor. Such functors are natural generalizations of the notion of a separable extension of rings. We provide the definitions and elementary facts about separable functors here for the benefit of the reader. We use these facts to prove Theorem B from the introduction.

Definition. Following [13] or [2], if $\mathcal{C}$ and $\mathcal{D}$ are arbitrary categories, a functor $F: \mathcal{C} \rightarrow \mathcal{D}$ is said to be separable if for each pair of objects $M, N \in \mathcal{C}$ there is a map $\phi: \operatorname{Hom}_{\mathcal{D}}(F M, F N) \rightarrow \operatorname{Hom}_{\mathcal{C}}(M, N)$ satisfying:

1. For all $\alpha \in \operatorname{Hom}_{\mathcal{C}}(M, N), \phi(F(\alpha))=\alpha$.

2. If there are $M^{\prime}, N^{\prime} \in \mathcal{C}$ and $\alpha \in \operatorname{Hom}_{\mathcal{C}}(M, N), \beta \in \operatorname{Hom}_{\mathcal{C}}\left(M^{\prime}, N^{\prime}\right), f \in$ $\operatorname{Hom}_{\mathcal{D}}\left(F M, F M^{\prime}\right), g \in \operatorname{Hom}_{\mathcal{D}}\left(F N, F N^{\prime}\right)$ such that the diagram

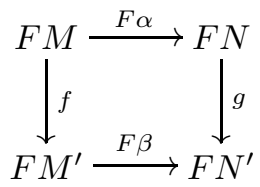


is commutative, then the diagram

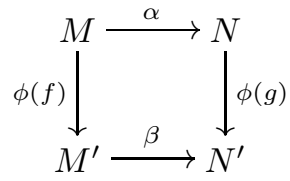

is also commutative.

If $\alpha: A \rightarrow B$ is a ring homomorphism, then the induction and restriction functors associated to $\alpha$ are defined as follows:

1. Restriction. Define ${ }_{\alpha}(-): B$-mod $\rightarrow A$-mod, which makes an $B$-module $M$ into an $A$-module ${ }_{\alpha}(M)$ via $a * m:=\alpha(a) m$.

2. Induction. Define $B \otimes_{A}-: A$-mod $\rightarrow B$-mod in the natural way.

Following [2], we say that $B / A$ is separable (or more precisely, the morphism $\alpha: A \rightarrow B$ is separable) provided the restriction functor ${ }_{\alpha}(-)$ is separable. This is the appropriate generalization of the classical definition of separable ring extensions in the commutative setting; see [3].

We record the following useful facts about separable functors below. We refer the reader to [2, Chapter 2] or [13] for the proofs.

Facts 2.1. Let $\mathcal{C}$ and $\mathcal{D}$ be abelian categories as above, let $F: \mathcal{C} \rightarrow \mathcal{D}$ be a separable functor, and let $M$ and $N$ be objects in $\mathcal{C}$. Let $\alpha: A \rightarrow B$ be a ring morphism. Then

1. [2, Proposition II.5.1.3] If $f: M \rightarrow N$ is a map in $\mathcal{C}$ such that $F(f)$ is split, then $f$ is split.

2. 2, Proposition II.5.1.3] If $F(M)$ is projective, then $M$ is projective.

3. 2, Proposition II.5.1.4] The restriction functor $\alpha(-)$ is separable if and only if the map $\Psi: B \otimes_{A} B \rightarrow B$ defined via $b \otimes b^{\prime} \mapsto b b^{\prime}$ splits as a $B$ - $B$-bimodule map.

4. 2, Proposition II.5.1.4] The induction functor $B \otimes_{A}-$ is separable if and only if $\alpha$ splits as an $A$-bimodule map.

In the particular situation where $A$ and $B$ are strongly graded rings, the induction and restriction functors are separable according to the following facts.

Proposition 2.2. Let $G$ be a finite group and let $B$ be $G$-strongly graded with 1component $B_{1}$. Let $H$ be a subgroup of $G$ and let $A$ be the truncation of $B$ at the subgroup $H$; that is, $A=\bigoplus_{h \in H} B_{h}$. Let $\alpha: A \rightarrow B$ be the inclusion map.

1. The induction functor $B \otimes_{A}-: A$-mod $\rightarrow B$-mod is separable.

2. If $H$ is normal and $|G: H|$ is a unit in $B_{1}$, then the restriction functor $\alpha(-): B$-mod $\rightarrow A$-mod is separable.

3. If $B$ is a crossed product, $B=\Delta *_{\tau}^{\alpha} G$, and $|G: H| \in\left(B_{1}\right)^{\bullet}$, then the restriction functor $\alpha(-): B$-mod $\rightarrow A-\bmod$ is separable.

Proof. (1) It is straightforward to see that there is an $A$-bimodule decomposition of $B$ as $B=A \oplus\left[\bigoplus_{g \in G \backslash H} B_{g}\right]$. Thus, $\alpha$ splits as an $A$ bimodule map, and so $B \otimes_{A}-$ is separable by Facts [2.1.

(2) This argument is analogous to the proof of Maschke's Theorem. Since $H$ is normal, $B$ is $G / H$-strongly graded; and so it suffices to prove the result when $H=1$. Let $|G|=n$. In view of statement (3) of Facts 2.1] we need to construct 
a $B$-bimodule map $\Phi: B \rightarrow B \otimes_{A} B$ so that $\Psi \Phi(b)=b$ for all $b \in B$. Since $B$ is $G$-strongly graded, we have that $B_{g^{-1}} \cdot B_{g}=B_{1}$ and so we can find, for each $g \in G$, elements $a\left(g^{-1}, i\right) \in B_{g^{-1}}$ and $b(g, i) \in B_{g}$, indexed over a finite set, such that $\sum_{i} a\left(g^{-1}, i\right) b(g, i)=1$.

Set $c=\sum_{g} \sum_{i} a\left(g^{-1}, i\right) \otimes b(g, i) \in B \otimes B$. We claim that $x c=c x$ for all $x \in B$. It suffices to prove this claim for the case when $x$ is homogeneous, so assume $x \in B_{y}$ for some $y \in G$. We observe that $b(g, i) x a\left(y^{-1} g^{-1}, k\right) \in B_{1}$, and so

$$
\begin{aligned}
c x & =\sum_{g} \sum_{i} a\left(g^{-1}, i\right) \otimes b(g, i) x \\
& =\sum_{g} \sum_{i} a\left(g^{-1}, i\right) \otimes b(g, i) x\left(\sum_{k} a\left(y^{-1} g^{-1}, k\right) b(g y, k)\right) \\
& =\sum_{g} \sum_{i} \sum_{k} a\left(g^{-1}, i\right) \otimes b(g, i) x a\left(y^{-1} g^{-1}, k\right) b(g y, k) \\
& =\sum_{g} \sum_{i} \sum_{k} a\left(g^{-1}, i\right) b(g, i) x a\left(y^{-1} g^{-1}, k\right) \otimes b(g y, k) \\
& =\sum_{g} \sum_{k} \sum_{i} a\left(g^{-1}, i\right) b(g, i) x a\left(y^{-1} g^{-1}, k\right) \otimes b(g y, k) \\
& =\sum_{g} \sum_{k} x a\left(y^{-1} g^{-1}, k\right) \otimes b(g y, k) \\
& =x \sum_{g} \sum_{k} a\left(y^{-1} g^{-1}, k\right) \otimes b(g y, k) \\
& =x c
\end{aligned}
$$

which proves the claim. Now define $\Phi: B \rightarrow B \otimes B$ via $\Phi(x)=n^{-1} c x$, which, by the claim, is a $B$-bimodule map. It follows that

$$
\Psi \Phi(x)=n^{-1} \sum_{g} \sum_{i} a\left(g^{-1}, i\right) b(g, i) x=n^{-1} \sum_{g} x=x
$$

as desired.

(3) We must show that $\Psi: B \otimes_{B_{H}} B \rightarrow B$ via $b_{1} \otimes b_{2} \mapsto b_{1} b_{2}$ is split as a $B$-bimodule homomorphism. In particular, we construct a $B$-bimodule map $\Phi$ : $B \rightarrow B \otimes_{A} B$ so that $\Psi \Phi(b)=b$ for all $b \in B$. Let $\{\bar{g} \mid g \in G\}$ denote the $\Delta$-basis for $B$. First observe that $\bar{g}^{-1} \otimes \bar{g}=\overline{h g}^{-1} \otimes \overline{h g}$ for all $h \in H$ and $g \in G$. This is because

$$
\begin{aligned}
\overline{h g}^{-1} \otimes \overline{h g} & =\bar{g}^{-1} \bar{h}^{-1} \tau(h, g) \otimes \tau(h, g)^{-1} \bar{h} \bar{g} \\
& =\bar{g}^{-1} \otimes \bar{h}^{-1} \tau(h, g) \tau(h, g)^{-1} \bar{h} \bar{g}=\bar{g}^{-1} \otimes \bar{g}
\end{aligned}
$$

Let $T$ denote the set of all right cosets of $H$, and set

$$
c=\sum_{H g \in T} \bar{g}^{-1} \otimes \bar{g}
$$

By the observation above, $c$ is independent of the choice of representatives of each coset. We claim that $c x=x c$ for all $x \in B$. First suppose $x=\bar{k}$, where $k \in G$. 
Then

$$
\begin{aligned}
c x & =\left(\sum_{H g \in T} \bar{g}^{-1} \otimes \bar{g}\right) \bar{k} \\
& =\sum_{H g \in T} \bar{g}^{-1} \otimes \bar{g} \bar{k} \\
& =\sum_{H g \in T} \overline{k k}^{-1} \bar{g}^{-1} \otimes \bar{g} \bar{k} \\
& =\bar{k} \sum_{H g \in T} \bar{k}^{-1} \bar{g}^{-1} \otimes \bar{g} \bar{k} \\
& =\bar{k} \sum_{H g \in T} \overline{g k}^{-1} \otimes \overline{g k} \\
& =\bar{k} \sum_{H g k \in T} \overline{g k}^{-1} \otimes \overline{g k} \\
& =\bar{k} c=x c .
\end{aligned}
$$

Now suppose $x \in \Delta$. Then

$$
\begin{aligned}
c x & =\left(\sum_{H g \in T} \bar{g}^{-1} \otimes \bar{g}\right) x \\
& =\left(\sum_{H g \in T} \bar{g}^{-1} \otimes \alpha(g)(x) \bar{g}\right) \\
& =\left(\sum_{H g \in T} \bar{g}^{-1} \alpha(g)(x) \otimes \bar{g}\right) \\
& =\left(\sum_{H g \in T} \alpha\left(g^{-1}\right)(\alpha(g)(x)) \bar{g}^{-1} \otimes \bar{g}\right) \\
& =x\left(\sum_{H g \in T} \bar{g}^{-1} \otimes \bar{g}\right) \\
& =x c .
\end{aligned}
$$

Since $B=\Delta * G, c$ commutes with all $x \in B$.

Now define $\Phi: B \rightarrow B \otimes B$ via $\Phi(x)=|G: H|^{-1} c x$, which, by the above, is a $B$-bimodule map. It follows that $\Psi \Phi(x)=|G: H|^{-1} \sum_{g} \bar{g}^{-1} \bar{g} x=n^{-1} \sum_{g} x=x$, as desired.

The converse of statement (2) of the above proposition is also true; see [11. Proposition 2.1 and Corollary 2.3].

Proposition 2.3. Let $A$ and $B$ be $R$-orders and suppose $F: A$-mod $\rightarrow B$-mod is a separable functor that takes $A$-lattices to $B$-lattices. If $B$ is hereditary, then $A$ is hereditary. In particular, if $B$ is a $G$-strongly graded, hereditary $R$-order and if $A=B_{H}$ for some subgroup $H$, then $A$ is hereditary.

Proof. If $I$ is a left ideal of $A$, then $F(I)$ is a $B$-lattice. Since $B$ is hereditary, $F(I)$ is projective. Consequently, $I$ is $A$-projective from Facts 2.1 and so $A$ is hereditary. For the second statement, use the separable induction functor $B \otimes_{A}-$.

We now have enough tools to prove Theorem B from the introduction and solve the hereditary problem for crossed products in the inner case. 
Theorem 2.4. Let $R$ denote a Dedekind ring whose quotient field $K$ is either a local or global field. Let $\Delta \neq R$ be a maximal $R$-order in a central semi-simple $K$-algebra, $G$ a group of order $n$, and $\alpha: G \rightarrow \operatorname{Inn}(\Delta)$ a homomorphism. For any factor set $\tau$ defined on $G$ with values in $R^{\bullet}$, the following statements are equivalent:

1. $\Delta *_{\tau}^{\alpha} G$ is maximal.

2. $\Delta *_{\tau}^{\alpha} G$ is hereditary.

3. $n=|G|$ is a unit of $R$.

Proof. Each of the three listed properties holds over a Dedekind ring $R$ if and only if it holds after completion at every prime. Hence it is sufficient to prove equivalence in case $R$ is a complete DVR.

$(1) \Rightarrow(2)$ is trivial.

$(2) \Rightarrow(3)$. Assume $\Delta * G$ is hereditary. It suffices to show that $p \in R^{\bullet}$ for each prime $p$ dividing $n$. Let $P$ be a $p$-Sylow subgroup of $G$ and choose an $x, e \neq x$, in $P$. Set $L=\langle x\rangle$. By Proposition [2.3, $\Delta * L$ is hereditary. Thus, we now assume that $G$ is cyclic, and we write $G=\langle\sigma\rangle$.

There is an element $u \in \Delta^{\bullet}$ with $\alpha(\sigma)=\iota_{u}$. Initially, write

$$
\Delta *_{\tau}^{\alpha} G=\sum_{j=0}^{n-1} \Delta w^{j}
$$

where $w \delta=\alpha(\sigma)(\delta) w=u \delta u^{-1} w$. Let $v=u^{-1} w$; then $v$ centralizes $\Delta$ and

$$
\Delta *_{\tau}^{\alpha} G=\sum_{j=0}^{n-1} \Delta v^{j}
$$

Now $u^{n}$ acts trivially by conjugation because $\sigma$ has order $n$; thus $u^{n}$ is in $R^{\bullet}$. Similarly $w^{n}$ is in $R^{\bullet}$, and it follows, using $u w=w u$, that $v^{n}=r \in R^{\bullet}$. It is now straightforward to verify there is an isomorphism

$$
\Delta \otimes \frac{R[X]}{\left(X^{n}-r\right)} \longrightarrow \Delta *_{\tau}^{\alpha} G
$$

determined by sending $X$ to $v$. This algebra is hereditary iff $R[X] /\left(X^{n}-r\right)$ is hereditary and unramified over $R[9]$. The extension is unramified only if the discriminant of the polynomial $\left(X^{n}-r\right)$ is a unit of $R$; the discriminant of $X^{n}-r$ is $\pm n^{n} r^{n-1}$. Thus the crossed product is hereditary only when $n$ is a unit of $R$.

$(3) \Rightarrow(1)$. By Proposition 2.2, we know that the restriction functor

$$
\mathcal{R}: \Delta * G-\bmod \rightarrow \Delta-\bmod
$$

is separable. Consequently, since $\Delta$ is maximal, $\Delta$ is hereditary, and so we have that $\Delta * G$ is hereditary by Proposition 2.3 .

Next we observe that if $M$ and $N$ are $\Delta * G$-modules such that $\mathcal{R}(M) \cong \mathcal{R}(N)$ as $\Delta$-modules, then $M \cong N$ as $\Delta * G$-modules. To see this, suppose $f: \mathcal{R}(M) \rightarrow \mathcal{R}(N)$ is an isomorphism. Define $\hat{f}: M \rightarrow N$ via $\hat{f}(m)=n^{-1} \sum_{x \in G} \bar{x} f\left(\bar{x}^{-1} m\right)$, where $\bar{x}$ denotes the basis element of $\Delta * G$ corresponding to $x \in G$. We claim that $\hat{f}$ is a 
left $\Delta * G$-homomorphism. If $y \in G$, then

$$
\begin{aligned}
\hat{f}(\bar{y} m) & =n^{-1} \sum_{x} \bar{x} f\left(\bar{x}^{-1} \bar{y} m\right) \\
& =n^{-1} \sum_{x} \bar{x} f\left(\tau\left(x^{-1}, y\right) \overline{x^{-1} y} m\right) \\
& =n^{-1} \sum_{x} \bar{x} \tau\left(x^{-1}, y\right) f\left(\overline{x^{-1} y} m\right) \\
& =n^{-1} \sum_{x} \overline{y y}{ }^{-1} \bar{x} \tau\left(x^{-1}, y\right) f\left(\overline{x^{-1} y} m\right) \\
& =n^{-1} \sum_{z} \bar{y} \bar{z} f\left(\bar{z}^{-1} m\right) \\
& =\bar{y} \hat{f}(m),
\end{aligned}
$$

where we make the substitution $\bar{z}^{-1}=\overline{x^{-1} y}$ so that $\bar{z}=\tau\left(x^{-1}, y\right) \bar{y}^{-1} \bar{x}$. On the other hand, if $d \in \Delta$, then

$$
\begin{aligned}
\hat{f}(d m) & =n^{-1} \sum_{x} \bar{x} f\left(\bar{x}^{-1} d m\right) \\
& =n^{-1} \sum_{x} \bar{x} \alpha\left(x^{-1}\right)(d) f\left(\bar{x}^{-1} m\right) \\
& =n^{-1} \sum_{x} \alpha(x)\left(\alpha\left(x^{-1}\right)(d)\right) \bar{x} f\left(\bar{x}^{-1} m\right) \\
& =n^{-1} \sum_{x} d \bar{x} f\left(\bar{x}^{-1} m\right) \\
& =d \hat{f}(m) .
\end{aligned}
$$

This proves the observation.

Now let $e$ be a central, primitive idempotent of $\Delta * G$. By [4, Theorem 26.20], it suffices to prove that $e(\Delta * G) e$ is maximal. Let $e_{1}$ and $e_{2}$ be two primitive idempotents of $\Delta * G$ such that $e_{i}=e e_{i}$ for $i=1,2$. Since $\Delta * G$ is hereditary over a complete, local Dedekind domain, it suffices to show that $(\Delta * G) e_{1} \cong(\Delta * G) e_{2}$ as left $\Delta * G$-lattices by the structure of hereditary orders (4 Theorem 26.28]). However, since $e(\Delta * G) e$ is prime, $K\left((\Delta * G) e_{1}\right) \cong K\left((\Delta * G) e_{2}\right)$. Since $\Delta$ is maximal, we see by [4, Exercise 11, p. 581] that $\mathcal{R}\left((\Delta * G) e_{1}\right) \cong \mathcal{R}\left((\Delta * G) e_{2}\right)$. By our observation above, $(\Delta * G) e_{1} \cong(\Delta * G) e_{2}$, and so the theorem is proved.

\section{MaXimal SUbFieldS}

Our goal in this section is to reduce to the case where the coefficient ring of the crossed product is a submatrix order with entries from a commutative ring. This suggests tensoring the division ring by a maximal subfield.

Notation 3.1. Let $R$ denote a complete DVR with maximal ideal $P=p R$ and quotient field $K$. Let $\Gamma$ denote a maximal $R$-order in a central simple $K$-algebra $A$. By [14] Theorem 17.3], there exist a positive integer $q$ and a maximal $R$-order $\Delta$ in a central division $K$-algebra $D$ such that $\Gamma=M_{q}(\Delta)$. Write $|D: K|=m^{2}$, so that the index of $D$ is $m$. Since the residue field $\bar{R}$ of $R$ is finite, there exists an inertia subfield $E$ of $D$ with index $m$; that is, $E$ is a maximal subfield of $D, E$ is unramified over $K,|E: K|=m$, and $E \otimes_{K} D \cong M_{m}(E)$. Let $S$ denote the integral closure of $R$ in $E$. 
We have $E \otimes_{S} S \otimes_{R} \Delta \cong E \otimes_{R} \Delta \cong\left(E \otimes_{K} K\right) \otimes_{R} \Delta \cong E \otimes_{K} D \cong M_{m}(E)$. Thus, $S \otimes_{R} \Delta$ embeds as an $S$-order inside the separable $E$-algebra $M_{m}(E)$. Similarly, $S \otimes_{R} \Gamma$ embeds as an $S$-order inside the separable $E$-algebra $M_{m q}(E)$.

Let $\alpha: G \rightarrow \operatorname{Aut}(\Delta)$ and $\beta: G \rightarrow \operatorname{Aut}(\Gamma)$ denote group homomorphisms and let $\tau: G \times G \rightarrow R \bullet$ be a factor set. Let $\Gamma * G$ denote $\Gamma *_{\tau}^{\beta} G$ and $\Delta * G$ denote $\Delta *_{\tau}^{\alpha} G$.

Remark 3.2. $S$ is unramified over $R$ by [14, p. 145].

Example 3.3. Let $R$ denote the complete 2-adic integers with prime ideal $2 R$ and quotient field $K$. Let $D$ denote the usual quaternion $K$-algebra $D=K(i, j, k)$ with $i^{2}=j^{2}=-1$ and $i j=k=-j i$. Let $\Delta$ denote the maximal $R$-order in $D$. By [14] p. 131], $\Delta=R[i, j, k]+R \alpha$, where $\alpha=(1+i+j+k) / 2$. Since $\alpha$ is a primitive cube root of $-1,-\alpha$ is a primitive cube root of 1 , and so the field extension $E=K(\alpha)$ is an inertia field of $D$ (see [14, p. 145] for details). Since this is unramified over $K$, the integral closure $S$ over $K$ in $E$ is unramified over $K$. Moreover, $S=R[\alpha]$ and $E \otimes D \cong M_{2}(E)$.

We want to make sure that $S \otimes\left(\Gamma *_{\tau}^{\alpha} G\right)$ is isomorphic to $(S \otimes \Gamma) *_{\tau^{\prime}}^{a^{\prime}} G$, where $\alpha^{\prime}$ and $\tau^{\prime}$ are the appropriate extensions of $\alpha$ and $\tau$ respectively.

Theorem 3.4. Assume Notation 3.1 holds.

1. The map $\alpha: G \rightarrow \operatorname{Aut}(\Gamma)$ and the factor set $\tau: G \times G \rightarrow \Gamma^{\bullet}$ extend to a map $\hat{\alpha}: G \rightarrow \operatorname{Aut}\left(S \otimes_{R} \Gamma\right)$ and to a factor set $\hat{\tau}: G \times G \rightarrow\left(S \otimes_{R} \Gamma\right)^{\bullet}$ in such a way that $\left(S \otimes_{R} \Gamma\right) *_{\hat{\tau}}^{\hat{\alpha}} G$ is a crossed product.

2. $S \otimes\left(\Gamma *_{\tau}^{\alpha} G\right) \cong\left(S \otimes_{R} \Gamma\right) *_{\hat{\tau}}^{\hat{\alpha}} G$ as graded rings.

Proof. (1) We first observe that since $\Gamma *_{\tau}^{\alpha} G$ is an $R$-order, then the center of $\Gamma * G$ contains $R$. Consequently, the map $\alpha: G \rightarrow \operatorname{Aut}(\Gamma)$ has an image that is contained in $\operatorname{Aut}_{R}(\Gamma)$, which are the $R$-automorphisms of $\Gamma$ (i.e., the automorphisms that fix $R$ elementwise). If $\phi \in \operatorname{Aut}_{R}(\Gamma)$, then $1_{S} \otimes_{R} \phi \in \operatorname{Aut}_{S}\left(S \otimes_{R} \Gamma\right)$, where $1_{S}$ denotes the identity automorphism of $S$. Thus, $\alpha$ extends to a map $\hat{\alpha}: G \rightarrow \operatorname{Aut}_{S}(S \otimes \Gamma)$ via the group homomorphism $G \rightarrow \operatorname{Aut}_{R}(\Gamma) \stackrel{1 \otimes(-)}{\longrightarrow} \operatorname{Aut}_{S}(S \otimes \Gamma)$.

Now if $u \in \Gamma^{\bullet}$, then $1 \otimes u \in(S \otimes \Gamma)^{\bullet}$, and so $\tau: G \times G \rightarrow(\Gamma)^{\bullet}$ extends to a map $\hat{\tau}: G \times G \rightarrow(S \otimes \Gamma)^{\bullet}$. We must show that $\hat{\tau}$ is a factor set for the map $\hat{\alpha}: G \rightarrow \operatorname{Aut}_{S}(S \otimes \Gamma)$. But

$$
\begin{aligned}
\hat{\tau}(x, y) \hat{\tau}(x y, z) & =(1 \otimes \tau(x, y))(1 \otimes \tau(x y, z)) \\
& =1 \otimes \tau(x, y) \tau(x y, z) \\
& =1 \otimes \alpha(x)(\tau(y, z)) \tau(x, y z) \\
& =(1 \otimes \alpha(g)(\tau(x, y)))(1 \otimes \tau(x, y z)) \\
& =\hat{\alpha}(x)(\hat{\tau}(x, y)) \hat{\tau}(x, y z),
\end{aligned}
$$

and so the associativity condition holds. We must also show that

$$
\hat{\alpha}(g)(\hat{\alpha}(h)(s \otimes d))=\hat{\tau}(g, h)(\hat{\alpha}(g h)(s \otimes d)) \hat{\tau}(g, h)^{-1} .
$$

But

$$
\begin{aligned}
\hat{\alpha}(g)(\hat{\alpha}(h)(s \otimes d)) & =s \otimes \alpha(g)(\alpha(h)(d)) \\
& =s \otimes \tau(g, h)(\alpha(g h) d) \tau(g, h)^{-1} \\
& =\hat{\tau}(g, h)(\hat{\alpha}(g h)(s \otimes d)) \hat{\tau}(g, h)^{-1} .
\end{aligned}
$$

Thus, $\hat{\tau}$ is a factor set relative to $\hat{\alpha}$. 
(2) By (1), we can form $\left(S \otimes_{R} \Gamma\right) *_{\hat{\tau}}^{\hat{\alpha}} G$, which is the set of finite sums of elements of the form $(s \otimes d) \overline{\bar{g}}$ where $s \in S, d \in \Gamma$ and $g \in G$. On the other hand, $S \otimes(\Gamma * G)$ consists of finite sums of elements of the form $s \otimes(d \bar{g})$ where $s \in S, d \in \Gamma$ and $g \in G$. The map $s \otimes(d \bar{g}) \mapsto(s \otimes d) \overline{\bar{g}}$ defines a graded ring isomorphism from $S \otimes \Gamma$ to $\left(S \otimes_{R} \Gamma\right) * G$. To see that this a ring homomorphism, note that $(s \otimes d \bar{g})(t \otimes e \bar{h})=$ $s t \otimes d \bar{g} e \bar{h}=s t \otimes d \alpha(g)(e) \tau(g, h) \overline{g h}$, which maps to $(s t \otimes d \alpha(g)(e) \tau(g, h)) \overline{\overline{g h}}=$ $(s \otimes d)(t \otimes \alpha(g)(e)) \tau(g, h) \overline{\overline{g h}}=(s \otimes d) \hat{\alpha}(g)(t \otimes e) \overline{\bar{g}} \overline{\bar{h}}=(s \otimes d) \overline{\bar{g}}(t \otimes e) \overline{\bar{h}}$.

The idea is to pass the hereditary problem from $\Gamma * G$ to $\left(S \otimes_{R} \Gamma\right) * G$. The key to this passage again involves the notion of separable functors. There is the natural ring inclusion $\alpha: \Gamma * G \rightarrow\left(S \otimes_{R} \Gamma\right) * G$, and subsequently we have the induction functor $\mathcal{I}=S \otimes_{R}-: \bmod -\Gamma \rightarrow \bmod -S \otimes \Gamma$ and the restriction functor $\mathcal{R}: \bmod -S \otimes \Gamma \rightarrow \bmod -\Gamma$. The point is that BOTH the induction and the restriction functors are separable, thanks to the unramified condition on $S$.

Theorem 3.5. Assume Notation 3.1 holds. Then $\Gamma * G$ is hereditary if and only if $(S \otimes \Gamma) * G$ is hereditary.

Proof. This is immediate from [9, Theorem 4].

So far we have been studying crossed products over maximal orders by finite groups. However, our technique will be to analyze the graded order $(S \otimes \Gamma) * G$ in place of the original order $\Gamma * G$. We observe that $(S \otimes \Gamma) * G$ is a crossed product over a hereditary but not maximal order in the next result.

Lemma 3.6. $S \otimes_{R} \Delta$ is a basic hereditary $S$-order inside $E \otimes D=M_{m}(E)$, but $S \otimes_{R} \Delta$ is not maximal unless $\Delta=R$.

Proof. The first statement follows from [9, Theorem 4]. It is basic because the type sequence for $S \otimes \Delta$ is $1, \ldots, 1$ ( $m$ times); see [9, Theorem 4 ] for details. For the second statement, we know, from [9, Theorem 7], that $S \otimes \Delta$ is maximal if and only if $\operatorname{gcd}\left(f_{S}, f_{R} m\right)=\operatorname{gcd}\left(f_{S}, f_{R}\right)$, where $f_{S}=|S / \operatorname{rad}(S): R / \operatorname{rad}(R)|$ and $f_{R}=|R / \mathrm{rad}(R): R / \mathrm{rad}(R)|=1$. But $f_{S}=|E: K|=m$, because $S$ is unramified over $R$ and $R$ is a complete DVR. Thus, $\operatorname{gcd}\left(f_{S}, f_{R} m\right)=m$, while $\operatorname{gcd}\left(f_{S}, f_{R}\right)=$ $\operatorname{gcd}(m, 1)=1$. Hence, $S \otimes_{R} \Delta$ is never maximal when $m>1$, or equivalently when $\Delta \neq R$.

We revisit the quaternion case.

Example 3.7. Assume the notation of Example 3.3 and let $P$ denote the maximal ideal of $S$. Since $S \otimes \Delta$ is hereditary but not maximal, it follows that $S \otimes \Delta$ is equal to $(\underset{P}{S} \underset{S}{S})$ for a suitable set of matrix units. Our work above indicates that $\Delta * G$ is hereditary if and only if $(\underset{P}{S} \underset{S}{S}) * G$ is hereditary.

Even though $S \otimes \Gamma$ is not maximal, we can still understand the representation type of $(S \otimes \Gamma) * G$ in certain situations. Having dealt with the inner case in section 2 , we turn our attention to the case in which $G$ acts as outer automorphisms of $\Gamma$. As a result, we investigate the $R$-automorphisms of $\Gamma$ and the $S$-automorphisms of $S \otimes \Gamma$, and, in particular, we prove that $G$ acts as outer automorphisms of $\Gamma$ if and only if $G$ acts as outer automorphisms of $S \otimes \Gamma$. 


\section{Automorphisms of $\Delta$ And $S \otimes \Delta$}

In this section, we assume Notation 3.1 holds and we analyze the $R$-automorphisms of $\Delta$ and the $S$-automorphisms of $S \otimes \Delta$.

Definition 4.1. Let $\Gamma$ denote any $R$-order and let $c(\Gamma)$ denote the center of $\Gamma$. An automorphism of $\Gamma$ that fixes $c(\Gamma)$ elementwise is called a central automorphism, and we denote the group of such automorphisms by Autcent $(\Gamma)$ or by $\operatorname{Aut}_{c(\Gamma)}(\Gamma)$. By automorphism, we mean a central automorphism.

For any ring $T$, we let $T^{\bullet}$ denote the units of $T$ and we denote an inner automorphism by $\iota_{u}$, where $u \in T^{\bullet}$ and $\iota_{u}(x)=u x u^{-1}$. Let $\operatorname{Inn}(T)$ denote the inner automorphisms of $T$.

Observe that every inner automorphism of $\Gamma$ is central, and so $\operatorname{Inn}(\Gamma) \subset$ Autcent $(\Gamma)$. An automorphism from Autcent $(\Gamma)$ is called a central outer automorphism if it is not inner; we denote the quotient group of central automorphisms modulo the inner automorphisms by $\operatorname{Outcent}(\Gamma)$; that is,

$$
\operatorname{Outcent}(\Gamma)=\operatorname{Autcent}(\Gamma) / \operatorname{Inn}(\Gamma) \text {. }
$$

The next result is an application of the Skolem-Noether theorem.

Lemma 4.2. If $\Gamma$ is an $R$-order in a central simple $K$-algebra, then

$$
\operatorname{Autcent}(\Gamma)=\left\{\sigma \in \operatorname{Aut}(\Gamma) \mid \exists u \in A^{\bullet} \text { such that } \sigma=\iota_{u}\right\} \text {. }
$$

Proof. If $\sigma \in \operatorname{Autcent}(\Gamma)$, then $1 \otimes_{R} \sigma \in \operatorname{Autcent}\left(K \otimes_{R} \Gamma\right)$, and $1 \otimes \sigma$ is inner by the Skolem-Noether Theorem. The converse is clear.

Our main goal for this section is to prove that $\operatorname{Outcent}(\Delta)$ and $\operatorname{Outcent}(S \otimes \Delta)$ are naturally isomorphic. We first analyze $\operatorname{Outcent}(\Delta)$.

Notation 4.3. Let $p$ denote the generator for the maximal ideal $P$ of $R$. By [14] Theorem 13.2], rad $\Delta$ is the unique maximal (1-sided and 2-sided) ideal of $\Delta$, and there is an element $\pi$ of $\Delta$ such that $\operatorname{rad} \Delta=\pi \Delta=\Delta \pi$. Since $\pi$ is a unit in $D$ and $\pi \Delta=\Delta \pi$, it follows that conjugation by $\pi$ defines a central automorphism of $\Delta$, which we denote by $\iota_{\pi}$. Using Notation 3.1 and [14, p. 145], the ramification index of $D$ over $K$ is $m$. Consequently, $p \Delta=(\operatorname{rad} \Delta)^{m}=\pi^{m} \Delta$.

Proposition 4.4. Outcent $(\Delta)$ is a cyclic group of order $m$ and is generated by $\operatorname{Inn}(\Delta) \cdot \iota_{\pi}$.

Proof. Every element of Outcent $(\Delta)$ is represented by an inner automorphism of $D$. If $v \in D^{\bullet}$, then $v=\delta / p^{k}$, where $\delta \in \Delta$ and $k$ is some nonnegative integer. Clearly, conjugation by $v$ equals conjugation by $\delta$. If $\delta \in \Delta^{\bullet}$, then $\iota_{v} \in \operatorname{Inn}(\Delta)$. Otherwise, we can write $\delta=w \pi^{\ell}$, where $w \in \Delta^{\bullet}$ and $\ell$ is some positive integer. Thus, $\operatorname{Inn}(\Delta) \iota_{v}=\operatorname{Inn}(\Delta) \iota_{\pi} \ell$. This shows that $\operatorname{Outcent}(\Delta)$ is generated by $\operatorname{Inn}(\Delta) \iota_{\pi}$. To see that the order of this group is $m$, note that $p=(\pi)^{m} w$, where $w$ is a unit of $\Delta$. Since $p$ is in the center of $\Delta,\left(\operatorname{Inn}(\Delta) \iota_{\pi}\right)^{m}=1$. Thus the order of $\iota_{\pi}$ is a divisor of $m$. If $\pi^{d} \in R$ for some $d$ with $0<d \leq m$, then $\pi$ is a root of an equation $X^{d}-r$ for some $r \in R$, and we have $|E: K|=m \leq d$ inasmuch as $E=K(\pi)$. Thus $d=m$.

Again we return to the concrete example of the quaternions. 
Example 4.5. Assume the notation from Examples 3.3 and 3.7 Since the ramification index of $D$ over $R$ is 2 , then $\operatorname{Outcent}(\Delta) \cong \mathbf{Z} / 2 \mathbf{Z}$ (the cyclic group of order 2 ) and is generated by $\iota_{\pi}$, where $\pi$ can be chosen to be $i-j$, for example. This is because $(i-j)^{2}=-2$ generates the maximal ideal of $R$.

Next we turn our attention to central automorphisms of $\Lambda$.

Notation 4.6. Since $\Lambda$ is a basic, hereditary $S$-order inside $M_{n}(E)$, the structure theorem for hereditary orders (14, Theorems 39.14 and 39.23]) implies that there is a ring isomorphism $\Phi: S \otimes \Delta \rightarrow \Lambda$, where $\Lambda$ is the matrix $S$-order

$$
\Lambda=\left(\begin{array}{ccccc}
S & S & \ldots & S & S \\
P & S & \ldots & S & S \\
\vdots & \vdots & & \vdots & \vdots \\
P & P & \ldots & P & S
\end{array}\right)
$$

Moreover, there exist precisely $n$ maximal orders $\Omega_{1}, \ldots, \Omega_{n}$ containing $\Lambda$, and $\Lambda=\bigcap^{n} \Omega_{i}$.

For $1 \leq i, j \leq n$, we use the notation $e(i, j)$ to denote the matrix basis element having 1 in the $(i, j)$-entry and zeros elsewhere. We will use $e_{i}$ to denote $e(i, i)$.

Lemma 4.7. Let $u$ be the matrix defined as $u=\sum_{i=2}^{n} e(i, i-1)+(1 / p) e(1, n)$. Then:

1. $u \in A^{\bullet}$, where $u^{-1}=\sum_{i=1}^{n-1} e(i, i+1)+p e(n, 1) \in \Lambda$.

2. The $\Lambda$-automorphism $\iota_{u}$ permutes the maximal orders $\Omega_{1}, \ldots, \Omega_{n}$; i.e., for $1 \leq i<n, \iota_{u}: \Omega_{i} \rightarrow \Omega_{i+1}$ and $\iota_{u}: \Omega_{n} \rightarrow \Omega_{1}$ are ring isomorphisms that preserve $\Lambda$.

3. For $1 \leq a \leq n, u^{a}=(1 / p) \sum_{z=1}^{a} e(z, n-a+z)+\sum_{y=a+1}^{n} e(y, y-a)$ and $\left(u^{-1}\right)^{a}=\sum_{x=1}^{n-a} e(x, x+a)+p \sum_{v=n-a+1}^{n} e(v, v+a-n)$.

4. For $1 \leq i, j, a \leq n$,

$$
\iota_{u^{a}}(e(i, j))= \begin{cases}e(i+a, j+a) & \text { if } 1 \leq i, j \leq n-a, \\ \frac{1}{p} e(i+a-n, j+a) & \text { if } n-a+1 \leq i \leq n \\ p e(i+a, j+a-n) & \text { and } 1 \leq j \leq n-a, \\ & \text { if } 1 \leq i \leq n-a \\ e(i+a-n, j+a-n) & \text { and } n-a+1 \leq j \leq n, \\ \text { if } n-a+1 \leq i, j \leq n .\end{cases}
$$

5. For $1 \leq i, a \leq n$,

$$
\iota_{u^{a}}\left(e_{i}\right)= \begin{cases}e_{i+a} & \text { if } i+a \leq n \\ e_{i+a-n} & \text { otherwise }\end{cases}
$$

Proof. (1) This is a straightforward verification.

(2) It is easily checked that $\iota_{u}$ maps $\Omega_{1}$ to

$$
\left(\begin{array}{ccccc}
S & P^{-1} & \ldots & P^{-1} & P^{-1} \\
P & S & \ldots & S & S \\
\vdots & \vdots & & \vdots & \vdots \\
P & S & \ldots & S & S
\end{array}\right)
$$

denote this as $\Omega_{2}$. Relabeling as necessary, we obtain that $\iota_{u}\left(\Omega_{j}\right)=\Omega_{j+1}$ for $1 \leq j \leq n-1$ and $\iota_{u}\left(\Omega_{n}\right)=\Omega_{1}$. Moreover, it is easily checked that $\Lambda$ is preserved. 
(3) We leave this computation to the reader.

(4) There are four cases to consider. First suppose $1 \leq j \leq n-a$. Then from (3), we have

$$
u^{a} e(i, j) u^{-a}=u^{a} e(i, j) e(j, j+a)=u^{a} e(i, j+a) .
$$

If $1 \leq i \leq n-a$, then $u^{a} e(i, j+a)=e(i+a, j+a)$, as claimed. If $n-a+1 \leq i \leq n$, then $u^{a} e(i, j+a)=(1 / p) e(i+a-n, j+a)$, as claimed.

Now suppose $n-a+1 \leq j \leq n$. Then, again from (3), we have

$$
u^{a} e(i, j) u^{-a}=u^{a} e(i, j) p e(j, j+a-n)=u^{a} e(i, j+a-n) p .
$$

If $1 \leq i \leq n-a$, then $u^{a} e(i, j+a-n) p=p e(i+a, j+a-n)$, while if $n-a+1 \leq i \leq n$, then $u^{a} e(i, j+a-n) p=p(1 / p) e(i+a-n, j+a-n)=e(i+a-n, j+a-n)$, as claimed.

(5) This follows directly from (4).

Definition 4.8. Let $\mathcal{O}$ denote the cyclic group of automorphisms of $\Lambda$ generated by the automorphism $\iota_{u}$ as defined in Lemma 4.7 Since $u^{n}=(1 / p) \cdot I$ and no lower power of $u$ is central, it follows that $\mathcal{O}$ has order $n$.

Lemma 4.9. If $\sigma \in \operatorname{Aut}(\Lambda)$ and $\sigma$ extends to $\sigma \in \operatorname{Aut}\left(\Omega_{j}\right)$ for some $j, 1 \leq j \leq n$, then $\sigma \in \operatorname{Inn}(\Lambda)$.

Proof. Since $S$ is a PID, it is well known that the $S$-automorphisms of $M_{n}(S)$ are inner; see, for example, 10]. Since all of the $\Omega_{j}$ 's are conjugate by [14, Theorem 17.3], every $S$-automorphism of $\Omega_{j}$ is inner for all $1 \leq j \leq n$. Thus, $\sigma=\iota_{z}$ for some $z \in\left(\Omega_{j}\right)^{\bullet}$. Using Lemma 4.7 let $\alpha \in A^{\bullet}$ be such that $\iota_{\alpha}: \Omega_{1} \rightarrow \Omega_{j}$ is a ring isomorphism that leaves $\Lambda$ invariant. Consequently, $\iota_{\alpha} \iota_{z} \iota_{\alpha^{-1}}=\iota_{\alpha z \alpha^{-1}}$ is an automorphism of $\Omega_{1}=M_{n}(S)$ that restricts to an automorphism of $\Lambda$. Thus, there is a unit $v$ of $\Omega_{1}$ such that $\iota_{\alpha z \alpha^{-1}}=\iota_{v}$. By [10, Proposition 1], $\iota_{\alpha z \alpha^{-1}}=\iota_{v}$ is actually inner on $\Lambda$, and so there exists a unit $v^{\prime} \in \Lambda^{\bullet}$ such that $\iota_{\alpha z \alpha^{-1}}=\iota_{v^{\prime}}$. It follows that $\iota_{z}=\iota_{\alpha^{-1} v^{\prime} \alpha}$ and $\alpha^{-1} v^{\prime} \alpha \in \Lambda^{\bullet}$. Thus, $\sigma$ is inner on $\Lambda$.

We arrive at the crux of our argument, namely, that Autcent $(\Lambda)$ is a semidirect product of $\operatorname{Inn}(\Lambda)$ and $\mathcal{O}$. This compares to Proposition 4.4.

Proposition 4.10. Autcent $(\Lambda)$ is the semidirect product of $\operatorname{Inn}(\Lambda)$ and $\mathcal{O}$, and $\mathcal{O} \cong \operatorname{Outcent}(\Lambda)$.

Proof. From Lemmas 4.7 and 4.9 $\mathcal{O}$ is disjoint from $\operatorname{Inn}(\Lambda)$. Clearly, $\Omega$ normalizes $\operatorname{Inn}(\Lambda)$ since $\operatorname{Inn}(\Lambda)$ is normal in $\operatorname{Aut}_{S}(\Lambda)$. Thus, we need only show that $\operatorname{Aut}_{S}(\Lambda)=$ $\operatorname{Inn}(\Lambda) \cdot \mathcal{O}$. But from the proof of [10, Theorem 2], $\operatorname{Aut}_{S}(\Lambda) / \operatorname{Inn}(\Lambda)$ is a cyclic group of order $n$. Thus, it follows that $\mathcal{O}$ generates $\operatorname{Aut}_{S}(\Lambda) / \operatorname{Inn}(\Lambda)$.

We next show that Outcent $(\Delta)$ and Outcent $(\Lambda)$ are naturally isomorphic. To describe these groups as subgroups of the Picard groups of $\Delta$ and $\Lambda$, we introduce some notation.

Definition 4.11. Let $\Omega$ denote an arbitrary $R$-order. The group $\operatorname{Picent}(\Omega)$ is the subgroup of $\operatorname{Pic}(\Omega)$ that consists of those invertible bimodules $\Omega M_{\Omega}$ that are centralized by the elements from $c(\Omega)$; that is, $c m=m c$ for all $c \in c(\Omega)$ and $m \in M$. It is well known that there is a group monomorphism $\Psi: \operatorname{Outcent}(\Omega) \rightarrow \operatorname{Picent}(\Omega)$ via $\phi \mapsto\left[\Omega_{\phi}\right]$, where $\Omega_{\phi}$ is the $\Omega$ - $\Omega$-bimodule that equals $\Omega$ as left module and has a right $\Omega$-action that is skewed by $\phi$. Moreover, $\Psi$ is an isomorphism if $\Omega$ is basic. See [14, Section 37] for details. 
Theorem 4.12. Let $\Phi: S \otimes \Delta \rightarrow \Lambda$ be the ring isomorphism from Notation 4.6 . Then:

1. Outcent $(\Delta) \cong \operatorname{Outcent}(\Lambda)$ via $\operatorname{Inn}(\Delta) \sigma \mapsto \operatorname{Inn}(\Lambda)\left(\Phi(1 \otimes \sigma) \Phi^{-1}\right)$. In particular,

$$
\operatorname{Inn}(\Delta)\left(\iota_{\pi}\right)^{\ell} \mapsto \operatorname{Inn}(\Lambda)\left(\iota_{u}\right)^{\ell}
$$

2. $\operatorname{Picent}(\Delta) \cong \operatorname{Picent}(\Lambda)$ via $\Delta_{\sigma} \mapsto \Lambda_{\Phi(1 \otimes \sigma) \Phi^{-1}}$

Proof. (1) We know that $\operatorname{rad} \Lambda=u^{-1} \Lambda=\Lambda u^{-1}$ and that $\operatorname{rad} \Lambda=\Phi(\operatorname{rad}(S \otimes \Delta))$. But

$$
\begin{aligned}
\operatorname{rad}(S \otimes \Delta) & =(\operatorname{rad} S) \otimes \Delta+S \otimes \operatorname{rad} \Delta \\
& =p S \otimes \Delta+S \otimes \pi \Delta \\
& =S \otimes p \Delta+S \otimes \pi \Delta \\
& =S \otimes \pi \Delta=(1 \otimes \pi)(S \otimes \Delta),
\end{aligned}
$$

since $S$ is unramified over $R$. Thus, $\operatorname{Inn}(\Lambda) \iota_{u}=\operatorname{Inn}(\Lambda) \iota_{\Phi(1 \otimes \pi)^{-1}}$.

Consequently,

$$
\operatorname{Inn}(\Lambda) \iota_{u}=\operatorname{Inn}(\Lambda) \iota_{\Phi\left(1 \otimes \pi^{-1}\right)}=\operatorname{Inn}(\Lambda) \Phi \iota_{1 \otimes \pi^{-1}} \Phi^{-1} .
$$

Now, since Outcent $(\Delta)$ is generated by $\iota_{\pi}{ }^{-1}$ with order $m$ and Outcent $(\Lambda)$ is generated by $\iota_{u}$ with order $m$, the proof is complete.

(2) Since both $\Delta$ and $\Lambda$ are basic, semiperfect rings, it follows that

$$
\operatorname{Outcent}(\Delta) \cong \operatorname{Picent}(\Delta) \text { via } \operatorname{Inn}(\Delta)\left(\iota_{\pi}\right)^{\ell} \mapsto \Delta_{\left(\iota_{\pi}\right)^{\ell}}
$$

and

$$
\operatorname{Outcent}(\Lambda) \cong \operatorname{Picent}(\Lambda) \text { via } \operatorname{Inn}(\Lambda)\left(\iota_{u}\right)^{\ell} \mapsto \Lambda_{\left(\iota_{u}\right)^{\ell}} .
$$

See 1 for details.

We use the above natural isomorphisms to show that $G$ acts as outer automorphisms on $\Delta$ if and only if $G$ acts as outer automorphisms on $S \otimes \Delta$, or, equivalently, on $\Lambda$.

Definition 4.13. Let $\Omega$ denote an arbitrary $R$-order. If $G$ is a finite group, then we say that $G$ acts on $\Omega$ as central outer automorphisms provided there is a group monomorphism $\alpha: G \rightarrow \operatorname{Autcent}(\Omega)$ such that $\alpha(G) \cap \operatorname{Inn}(\Omega)=1$.

Theorem 4.14. The group $G$ acts as central outer automorphisms on $\Delta$ via $\alpha$ : $G \rightarrow \operatorname{Autcent}(\Delta)$ if and only if $G$ acts as central outer automorphisms on $\Lambda$ via $\alpha^{\prime}=\Phi(1 \otimes \alpha(-)) \Phi^{-1}: G \rightarrow \operatorname{Aut}(\Lambda)$.

Proof. $(\Rightarrow)$ Suppose $G$ acts as central outer automorphisms on $\Delta$ so that there is a group monomorphism $\alpha: G \rightarrow \operatorname{Autcent}(\Delta)$ such that $\alpha(G) \cap \operatorname{Inn}(\Delta)=1$. We show that $\alpha^{\prime}=\Phi(1 \otimes \alpha(-)) \Phi^{-1}: G \rightarrow \operatorname{Aut}(\Lambda)$ is an outer action of $G$ on $\Lambda$. But if $g \in G \backslash\{1\}$, then $\alpha(g) \notin \operatorname{Inn}(\Delta)$, and so, by Theorem 4.12, $\alpha^{\prime}(g) \notin \operatorname{Inn}(\Lambda)$. So $G$ acts as central outer automorphisms on $\Lambda$ via $\alpha^{\prime}$.

$(\Leftarrow)$ Suppose $\alpha^{\prime}=\Phi(1 \otimes \alpha(-)) \Phi^{-1}: G \rightarrow \operatorname{Aut}(\Lambda)$ is an outer action. But by Theorem 4.12, it follows immediately that $\alpha: G \rightarrow \operatorname{Autcent}(\Delta)$ is outer.

Combining Theorems 3.4, 3.5 and 4.14, we have the final result of this section.

Corollary 4.15. $G$ acts as central outer automorphisms on $\Delta$ and $\Delta *_{\tau}^{\alpha} G$ is hereditary if and only if $G$ acts as central outer automorphisms on $\Lambda$ and $\Lambda *_{\tau^{\prime}}^{\alpha^{\prime}} G$ is hereditary. 


\section{THE OUTER CASE FOR $\Lambda$}

We now consider $\Lambda * G$, where $G$ acts on $\Lambda$ as a set of central outer automorphisms of $\Lambda$, and we show that $\Lambda * G$ is a hereditary order. See Theorem 5.13 Our first result is a change of basis so that we may assume $G$ is a subgroup of $\mathcal{O}$. Throughout this section, we assume Notation 4.6 holds.

Theorem 5.1. Let $G$ be a finite group and let $T$ be an $S$-order. Let $\alpha: G \rightarrow$ Aut $(T)$ be a central outer action of $G$ on $T$ and let $\tau$ denote a factor set. Assume that Autcent $(T)$ is a semidirect product of $\operatorname{Inn}(T)$ and a cyclic subgroup $H$ of order n. Then:

1. $G$ is cyclic of order $d$ which divides $n$.

2. $\alpha$ is a group monomorphism that extends to a group monomorphism $\alpha: G \rightarrow$ $\operatorname{Out}(T)$.

3. There exist a group monomorphism $\alpha^{\prime}: G \rightarrow H$, a factor set $\tau^{\prime}$, and a change in basis so that $T *_{\tau}^{\alpha} G=T *_{\tau^{\prime}}^{\alpha^{\prime}} G$.

Proof. (1) and (2). The values of $\tau$ are in $S^{\bullet}$; so, for any $x, y \in G$ and $\lambda \in T$, $\alpha(x)(\alpha(y)(\lambda)):=\tau(x, y)[\alpha(x y)(\lambda)] \tau(x, y)^{-1}=\alpha(x y)(\lambda)$, and so $\alpha$ is a monic group homomorphism. Since $G$ acts as outer automorphisms, $\alpha$ extends to a monomorphism $\alpha: G \rightarrow \operatorname{Aut}(T) \rightarrow \operatorname{Out}(T)$. Since $\operatorname{Out}(T) \cong H$ and $H$ is cyclic of order $n, G$ is isomorphic to a cyclic subgroup of $H$. Consequently, $G$ must have order $d$ that divides $n=|H|$. Let $m=n / d$.

(3) Let $g$ be a generator for $G$; use the $T$-basis $1, \bar{g}, \overline{g^{2}}, \ldots, \overline{g^{d-1}}$ of $\Gamma$ as determined by $G$. Let $H=\langle h\rangle$ and let $w=u^{m}$ be such that $\langle w\rangle$ is the subgroup of order $d$ inside $H$. Since $\alpha$ extends to a group monomorphism $\alpha: G \rightarrow \operatorname{Outcent}(T) \cong H$, $\alpha(g)$ and $\iota_{w}$ differ by an inner automorphism of $T$. Consequently, there exists $v_{1} \in T^{\bullet}$ such that $\alpha(g)=\iota_{v_{1} w}$. Let $v_{0}=1$.

We claim that $\alpha\left(g^{i}\right)=\iota_{v_{i} w^{i}}$, where

$$
v_{i}=v_{1} \iota_{w}\left(v_{1}\right) \iota_{w^{2}}\left(v_{1}\right) \ldots \iota_{w^{i-1}}\left(v_{1}\right)=v_{1}\left({ }^{w} v_{1}\right)\left({ }^{w^{2}} v_{1}\right) \ldots\left({ }^{w^{i-1}} v_{1}\right)
$$

where $1 \leq i \leq d$. (Here ${ }^{w} v$ denotes the element $\iota_{w}(v)$.) But since $\alpha$ is a group homomorphism, we have

$$
\begin{aligned}
\alpha\left(g^{i}\right) & =\alpha(g) \ldots \alpha(g) \\
& =\iota_{v_{1} w} \ldots \iota_{v_{1} w} \\
& =\iota_{v_{1} w v_{1} w \ldots v_{1} w}
\end{aligned}
$$

and $v_{1} w v_{1} w \ldots v_{1} w=v_{1}\left({ }^{w} v_{1}\right)\left(w^{2} v_{1}\right) \ldots\left({ }^{w^{i-1}} v_{1}\right) w^{i}$, so the claim is proved.

For $1 \leq i \leq d-1$, define $\overline{\overline{g^{i}}}:=v_{i}{ }^{-1} \overline{g^{i}}$. We will show that $1, \overline{\bar{g}}, \overline{\overline{g^{2}}}, \ldots, \overline{\overline{g^{d-1}}}$ is the desired change of basis. Since each $v_{i}$ is a unit of $T$, it follows that $T \cdot \overline{g^{i}}=T \cdot \overline{\overline{g^{i}}}$. Thus, with this new basis, we need only show that we obtain the appropriate group action $\alpha^{\prime}$ and twisting $\tau^{\prime}$. We claim that conjugation by $\overline{\overline{g^{j}}}$ gives rise to the automorphism $\iota_{w^{j}}$ of $T$. To see this, note that

$$
\begin{aligned}
\overline{\overline{g^{j}}} \lambda\left(\overline{\overline{g^{j}}}\right)^{-1} & =v_{j}{ }^{-1} \overline{g^{j}} \lambda \overline{g^{j}}-1 \\
& =v_{j}{ }^{-1} \alpha\left(g^{j}\right)(\lambda) \overline{g^{j}}\left(\overline{g^{j}}\right)^{-1} v_{j} \\
& =v_{j}{ }^{-1} \iota_{v_{j} w^{j}}(\lambda) v_{j} \\
& =\iota_{w^{j}}(\lambda) .
\end{aligned}
$$


It follows that there is a group monomorphism $\alpha^{\prime}: G \rightarrow H$ via $\alpha^{\prime}\left(g^{j}\right)=\iota_{w^{j}}$, and $\overline{\overline{g^{j}}} \lambda=\alpha^{\prime}\left(g^{j}\right)(\lambda) \overline{\overline{g^{j}}}$ for all $\lambda \in T$.

Next we identify the factor set $\tau^{\prime}$ associated to the action $\alpha^{\prime}: G \rightarrow H$. We have already observed (using Lemma 4.7) that $u^{n}=(1 / p) \cdot I$, and so $\iota_{u^{n}}=\iota_{w^{d}}=$ 1. Consequently, we can show that $v_{d} \in S^{\bullet}$. Specifically, since $\alpha$ is a group homomorphism, $\alpha\left(g^{d}\right)=1$, and so

$$
\begin{aligned}
\lambda=\alpha\left(g^{d}\right)(\lambda) & =\iota_{v_{d} w^{d}}(\lambda) \\
& =v_{d} w^{d} \lambda\left(w^{d}\right)^{-1} v_{d}^{-1} \\
& =v_{d} u^{n} \lambda u^{-n} v_{d}^{-1} \\
& =v_{d} \lambda v_{d}^{-1} .
\end{aligned}
$$

Hence, $v_{d} \in Z(T)=S$.

We claim that

$$
\alpha\left(g^{i}\right)\left(v_{j}\right)= \begin{cases}v_{i+j} v_{i}^{-1} & \text { if } i+j<d \\ v_{d} v_{i+j-d} v_{i}^{-1} & \text { otherwise }\end{cases}
$$

Note that

$$
\begin{aligned}
\alpha\left(g^{i}\right)\left(v_{j}\right) & =\alpha\left(g^{i}\right)\left(v_{1}\left({ }^{w} v_{1}\right)\left({ }^{w^{2}} v_{1}\right) \ldots\left({ }^{w^{j-1}} v_{1}\right)\right) \\
& =v_{i} \iota_{w^{i}}\left(v_{1}\left({ }^{w} v_{1}\right)\left({ }^{w^{2}} v_{1}\right) \ldots\left({ }^{w^{j-1}} v_{1}\right)\right) v_{i}{ }^{-1} \\
& =v_{i}\left({ }^{w^{i}} v_{1}\left({ }^{w^{i+1}} v_{1}\right)\left({ }^{w^{i+2}} v_{1}\right) \ldots\left({ }^{w^{i+j-1}} v_{1}\right)\right) v_{i}{ }^{-1} \\
& =\left(v_{1}\left({ }^{w} v_{1}\right)\left({ }^{w^{2}} v_{1}\right) \ldots\left({ }^{w^{i+j-1}} v_{1}\right)\right) v_{i}{ }^{-1} \\
& = \begin{cases}v_{i+j} v_{i}{ }^{-1} & \text { if } i+j<d, \\
v_{d} v_{i+j-d} v_{i}{ }^{-1} & \text { if } i+j \geq d .\end{cases}
\end{aligned}
$$

Define $\tau^{\prime}: G \times G \rightarrow S^{\bullet}$ via

$$
\tau^{\prime}\left(g^{i}, g^{j}\right)= \begin{cases}\tau\left(g^{i}, g^{j}\right) & \text { if } i+j<d \\ v_{d}{ }^{-1} \tau\left(g^{i}, g^{j}\right) & \text { if } i+j \geq d\end{cases}
$$

We show that $\tau^{\prime}$ is the appropriate factor set. First we show that $\overline{\overline{g^{i}}} \cdot \overline{\overline{g^{j}}}=$ $\tau^{\prime}\left(g^{i}, g^{j}\right) \overline{\overline{g^{i+j}}}$. We have

$$
\begin{aligned}
\overline{\overline{g^{i}}} \cdot \overline{\overline{g^{j}}} & =v_{i}{ }^{-1} \overline{g^{i}} v_{j}{ }^{-1} \overline{g^{j}} \\
& =v_{i}^{-1} \alpha\left(g^{i}\right)\left(v_{j}^{-1}\right) \tau\left(g^{i}, g^{j}\right) \overline{g^{i+j}} \\
& =v_{i}^{-1} \alpha\left(g^{i}\right)\left(v_{j}\right)^{-1} \tau\left(g^{i}, g^{j}\right) \overline{g^{i+j}} \\
& = \begin{cases}v_{i}^{-1} v_{i} v_{i+j}{ }^{-1} \tau\left(g^{i}, g^{j}\right) \overline{g^{i+j}} & \text { if } i+j<d, \\
v_{i}{ }^{-1} v_{i} v_{i+j-d} v_{d}^{-1} \tau\left(g^{i}, g^{j}\right) \overline{g^{i+j}} & \text { if } i+j \geq d,\end{cases} \\
& = \begin{cases}\tau\left(g^{i}, g^{j}\right) \overline{\overline{g^{i+j}}} & \text { if } i+j<d, \\
v_{d}{ }^{-1} \tau\left(g^{i}, g^{j}\right) \overline{\overline{g^{i+j}}} & \text { if } i+j \geq d,\end{cases} \\
& =\tau^{\prime}\left(g^{i}, g^{j}\right) \overline{\overline{g^{i+j}}}
\end{aligned}
$$

It remains to show that $\tau^{\prime} \in H^{2}\left(G, S^{\bullet}\right)$. However, this is a tedious, case-by-case verification which we leave to the reader. 
Corollary 5.2. Let $\alpha: G \rightarrow \operatorname{Aut}(\Lambda)$ be a central outer action of $G$ on $\Lambda$ and let $\tau$ denote a factor set. Then:

1. $G$ is cyclic of order $d$ which divides $n$.

2. $\alpha$ is a group monomorphism that extends to a group monomorphism $\alpha: G \rightarrow$ $\operatorname{Out}(\Lambda)$.

3. There exist a group monomorphism $\alpha^{\prime}: G \rightarrow \mathcal{O}$, a factor set $\tau^{\prime}$, and a change in basis so that $\Lambda *_{\tau}^{\alpha} G=\Lambda *_{\tau^{\prime}}^{\alpha^{\prime}} G$.

Proof. By Proposition 4.10 (i.e., Autcent $(\Lambda)$ is the semidirect product of $\operatorname{Inn}(\Lambda)$ and $\mathcal{O}$ ), the hypotheses of Theorem 5.1 are satisfied.

The above result now allows us to assume that we have a faithful group action $\alpha: G \rightarrow \mathcal{O}$ with associated cocyle $\tau \in H^{2}\left(G, S^{\bullet}\right)$, and that $g^{i}$ acts on $\Lambda$ via $\iota_{w^{i}}$.

For notational simplicity, let $e_{i}$ denote the matrix $e(i, i)$ and let $\Gamma=\Lambda * G=$ $\Lambda *_{\tau}^{\alpha} G$. By Lemma 4.7 we know that

$$
\alpha\left(g^{j}\right)\left(e_{i}\right)= \begin{cases}e_{i+m j} & \text { if } i+m j \leq n, \\ e_{i+m j-n} & \text { otherwise. }\end{cases}
$$

Consequently $G$ permutes the $e_{i}$, and so $G$ permutes the elements $1, \ldots, n$. Let ${ }^{x} i$ denote this action on $i$ by an element $x \in G$. Let ${ }^{G} i$ denote the orbit of $i$ under $G$.

As in the proof of Theorem [5.1, we let $1, \bar{g}, \overline{g^{2}}, \ldots, \overline{g^{d-1}}$ denote a basis for $\Gamma$.

The next result identifies the isomorphism classes of the projective indecomposable modules of $\Gamma$.

Lemma 5.3. For each $1 \leq i, j \leq n$,

$$
e_{i} \Gamma e_{j} \cdot e_{j} \Gamma e_{i}= \begin{cases}e_{i} \Gamma e_{i}, & j \in{ }^{G} i, \\ p \cdot e_{i} \Gamma e_{i}, & j \notin{ }^{G} i\end{cases}
$$

In particular, $e_{i} \Gamma \cong e_{j} \Gamma$ if and only if $j \in{ }^{G} i$.

Proof. Suppose $j={ }^{x} i$, where $x \in G$. Then it is clear that

$$
\left(e_{j} \bar{x}\right)\left(e_{i} \Gamma\right)=e_{j} e_{j} \bar{x} \Gamma=e_{j} \Gamma
$$

which shows that $e_{i} \Gamma \cong e_{j} \Gamma$. It follows that $e_{j} \Gamma e_{i} \cdot e_{i} \Gamma e_{j}=e_{j} \Gamma e_{j}$ and $e_{i} \Gamma e_{j} \cdot e_{j} \Gamma e_{i}=$ $e_{i} \Gamma e_{i}$. This proves one direction.

Assume that $j \notin{ }^{G} i$ and suppose, by way of contradiction, that $e_{j} \Gamma e_{i} \cdot e_{i} \Gamma e_{j}=$ $e_{j} \Gamma e_{j}$. Let $z \in e_{j} \Gamma e_{j} \cap \Lambda$. We can write $\sum a_{u} b_{u}=z$, where $a_{u} \in e_{j} \Gamma e_{i}$ and $b_{u} \in e_{i} \Gamma e_{j}$. We consider just one term of this sum, and label it $a b$, where $a \in e_{j} \Gamma e_{i}$ and $b \in e_{i} \Gamma e_{j}$. Write $a=\sum_{x \in G} e_{j} \lambda_{x} \bar{x} e_{i}$ and $b=\sum_{y \in G} e_{i} \lambda_{y} \bar{y} e_{j}$, where $\lambda_{x}, \lambda_{y} \in \Lambda$. Then

$$
a b=\sum_{x, y \in G} e_{j} \lambda_{x} \bar{x} e_{i} \lambda_{y} \bar{y} e_{j}=\sum_{x, y \in G} e_{j} \lambda_{x} e_{x} \alpha(x)\left(\lambda_{y}\right) \tau(x, y) \overline{x y} e_{j} .
$$

Now since $z$ is homogeneous inside $\Lambda$, the above terms for which $x y$ is not the identity must be zero. Hence the right side becomes

$$
a b=\sum_{x, y \in G, x y=1} e_{j} \lambda_{x} e_{x} \alpha(x)\left(\lambda_{y}\right) e_{j} .
$$

Since $j \neq{ }^{x} i$, either $j>{ }^{x} i$ or ${ }^{x} i>j$. In the former case, using the structure of $\Lambda, e_{j} \lambda_{x} e_{x_{i}}$ must be divisible by $p$. In the latter case, $e_{x}{ }^{x} \lambda_{y} e_{j}$ is divisible by $p$. Consequently, each term in the sum $\sum a_{u} b_{u}$ is divisible by $p$, and so $z \in p\left(e_{j} \Gamma e_{j}\right)$. Now any other homogeneous element of $e_{j} \Gamma e_{j}$ can be written as $z \bar{x}$, where $z \in$ 
$e_{j} \Gamma e_{j} \cap \Lambda$ and $x \in G$. As this element must also be divisible by $p$, we have $e_{i} \Gamma e_{j}$. $e_{j} \Gamma e_{i} \subseteq p e_{i} \Gamma e_{j}$. Next we show that the right side is contained in the left side.

It suffices to prove that $p e_{i}$ belongs to the left side, because the left side is an $e_{i} \Gamma e_{i}$-bimodule. (That is, $p e_{i} \Gamma e_{i}=\left(p e_{i}\right)\left(e_{i} \Gamma e_{i}\right) \subset e_{i} \Gamma e_{j} \cdot e_{j} \Gamma e_{i}$.) Since $j$ is not in the orbit of $i$, then either $i>j$ or $i<j$. In the former case, pe $(i, j)$ and $e(j, i)$ belong to $\Lambda$ and so to $\Gamma$. Thus, $p e_{i}=p e(i, j) e(j, i)$ belongs to $e_{i} \Gamma e_{j} * e_{j} \Gamma e_{i}$. If $i<j$ then $p e(j, i)$ and $e(i, j)$ belong to $\Lambda$ and to $\Gamma$. Thus, $p e_{i}=e(i, j) p e(j, i)$ belongs to $e_{i} \Gamma e_{j} * e_{j} \Gamma e_{i}$. The proof is complete.

Next we identify $e_{i} \Gamma e_{i}$.

Theorem 5.4. Let $v=\prod_{i=1}^{d-1} \tau\left(g^{i}, g\right) \in S^{\bullet}$. For any $1 \leq i \leq n$ such that $i$ is the smallest positive integer in the orbit ${ }^{G} i$, we have

$$
e_{i} \Gamma e_{i} \cong \frac{S[X]}{\left\langle X^{d}-p v\right\rangle}=S[z]
$$

where $z^{d}=p v$. Moreover, $e_{i} \Gamma e_{i}$ is a $G$-graded ring with $S$-basis $\left\{e\left(i,{ }^{x} i\right) \cdot \bar{x} \mid x \in G\right\}$.

Proof. Let $\gamma \in \Gamma$ and write $\gamma=\sum_{x \in G} \gamma_{x} \bar{x}$ with $\gamma_{x} \in \Lambda$. Then

$$
\begin{aligned}
e_{i} \gamma e_{i} & =\sum_{x \in G} e_{i} \gamma_{x} \bar{x} e_{i} \\
& =\sum_{x \in G} e_{i} \gamma_{x} \alpha(x)\left(e_{i}\right) \bar{x} \\
& =\sum_{x \in G} e_{i} \gamma_{x} e_{x} \bar{x} \\
& =\sum_{x \in G} e\left(i,{ }^{x} i\right) s\left(i,{ }^{x} i, x\right) \bar{x}
\end{aligned}
$$

where the $\left(i,{ }^{x} i\right)$-entry of $\gamma_{x}$ is $s\left(i,{ }^{x} i, x\right) \in S$. This shows that, as $S$-module, $e_{i} \Gamma e_{i}$ is spanned by the elements $e\left(i,{ }^{x} i\right) \cdot \bar{x}$; that is,

$$
e_{i} \Gamma e_{i}=\sum_{x \in G} S \cdot e\left(i,{ }^{x} i\right) \cdot \bar{x}
$$

Moreover, this sum is direct. To see this, note that

$$
S \cdot e\left(i,{ }^{x} i\right) \cdot \bar{x} \cap \sum_{y \neq x \in G} S \cdot e\left(i,{ }^{y} i\right) \cdot \bar{y} \subset \Lambda \cdot \bar{x} \cap\left(\sum_{y \neq x \in G} \Lambda \cdot \bar{y}\right) .
$$

The latter quantity is 0 since $\Gamma=\Lambda * G$ is graded by $G$. Finally, we can grade $e_{i} \Gamma e_{i}$ by the components $S e\left(i,{ }^{x} i\right) \bar{x}$. Note that for $x, y \in G$,

$$
\begin{aligned}
S e\left(i,{ }^{x} i\right) \bar{x} \cdot S e\left(i,{ }^{y} i\right) \bar{y} & =S e\left(i,{ }^{x} i\right) \alpha(x)\left(e\left(i,{ }^{y} i\right)\right) \overline{x y} \\
& \subset S e\left(i,{ }^{x} i\right) e\left({ }^{x} i,{ }^{x y} i\right) \overline{x y} \\
& =S e\left(i,{ }^{x y} i\right) \overline{x y},
\end{aligned}
$$

so that the multiplication is graded. However, the grading is not strong.

Since the elements of $G$ are $1, g, g^{2}, \ldots, g^{d-1}$, we can write the basis elements as powers of $\bar{g}$. That is, for $0 \leq j \leq d-1,(\bar{g})^{j}=\tau(g, g) \cdot \tau\left(g^{2}, g\right) \ldots \tau\left(g^{j-1}, g\right) \cdot \overline{g^{j}}=$ $t_{j} \cdot \overline{g^{j}}, t_{j} \in R^{\bullet}$, and so

$$
S \cdot e\left(i,{ }^{g^{j}} i\right) \cdot\left(\overline{g^{j}}\right)=S \cdot e\left(i,{ }^{g^{j}} i\right) \cdot t_{j}^{-1} \cdot \overline{g^{j}}=S \cdot e\left(i,{ }^{g^{j}} i\right) \cdot(\bar{g})^{j} .
$$


Consequently, we can write $e_{i} \Gamma e_{i}=\bigoplus_{j=0}^{d-1} S \cdot e\left(i, g^{j} i\right)(\bar{g})^{j}$. The identity element is $e(i, i) \overline{1}$.

Define the map $\psi: S[X] \rightarrow e_{i} \Gamma e_{i}$ via $\sum s_{j} X^{j} \mapsto \sum s_{j} e_{i, g^{j}} \bar{g}^{j}$. This is easily seen to be a surjective ring homomorphism. We wish to identify ker $\psi$. We claim that $\left(e\left(i,{ }^{g} i\right) \cdot \bar{g}\right)^{d}=v \cdot p \cdot e(i, i) \overline{1}$. To see this, we need to be more explicit about the action of $g^{j}$ on $e\left(i, g^{k} i\right)$.

Since $i$ is the smallest positive integer in ${ }^{G} i$ and since $\alpha(g)=\iota_{u^{m}}$, we have that

$$
\begin{aligned}
i \leq m & <{ }^{g} i=i+m \leq 2 m<{ }^{g^{2}} i=i+2 m \leq 3 m<\ldots \\
& <{ }^{g^{d-1}} i=i+(d-1) m \leq d m=n .
\end{aligned}
$$

This fact uses Lemma 4.7 repeatedly. Consequently, the basis elements for $e_{i} \Gamma e_{i}$ take on the form $e(i, i+m j) \bar{g}^{j}$. These are powers of $e(i, i+m) \bar{g}$ by the grading. Now we are ready to show that $(e(i, i+m) \bar{g})^{d}=p \cdot v e(i, i)$. Using Lemma 4.7 and $(*)$, we first note that, for $0 \leq j \leq d-2$,

$$
\alpha\left(g^{j}\right)(e(i, i+m))=\iota_{u^{m j}}(e(i, i+m))=e(i+m j, i+m(j+1)),
$$

while

$$
\begin{aligned}
\alpha\left(g^{d-1}\right)(e(i, i+m)) & =\iota_{u^{m(d-1)}}(e(i, i+m)) \\
& =p \cdot e(i+m(d-1), i+m(d)-n) \\
& =p \cdot e(i+m(d-1), i) .
\end{aligned}
$$

This follows from Lemma 4.7 by letting $a=m(d-1)$, so that $1 \leq i \leq m=n-a$ and $m+1=n-a+1 \leq i+m \leq n$.

Consequently,

$$
\begin{aligned}
(e(i, i+m) \cdot \bar{g})^{d}= & (e(i, i+m) \bar{g})(e(i, i+m) \bar{g})(e(i, i+m) \bar{g})^{d-2} \\
= & e(i, i+m) \alpha(g)(e(i, i+m)) \tau(g, g) \overline{g^{2}}(e(i, i+m) \bar{g})^{d-2} \\
= & \tau(g, g) e(i, i+m) e(i+m, i+2 m) \overline{g^{2}}(e(i, i+m) \bar{g})^{d-2} \\
= & \tau(g, g) e(i, i+2 m) \alpha\left(g^{2}\right)(e(i, i+m)) \tau\left(g^{2}, g\right) \\
& \cdot g^{3}(e(i, i+m) \bar{g})^{d-3} \\
= & \tau(g, g) \tau\left(g^{2}, g\right) e(i, i+2 m) e(i+2 m, i+3 m) \\
& \quad \overline{g^{3}}(e(i, i+m) \bar{g})^{d-3} \\
= & \tau(g, g) \tau\left(g^{2}, g\right) e(i, i+3 m) \overline{g^{3}}(e(i, i+m) \bar{g})^{d-3} \\
= & \ldots \\
= & \tau(g, g) \tau\left(g^{2}, g\right) \ldots \tau\left(g^{d-2}, g\right) \\
= & \tau(g, g) \tau\left(g^{2}, g\right) \ldots \tau\left(g^{d-2}, g\right) e(i, i+(d-1) m) \\
& \cdot \alpha\left(g^{d-1}\right)(e(i, i+m)) \tau\left(g^{d-1}, g\right) \overline{g^{d}} \\
= & \tau(g, g) \tau\left(g^{2}, g\right) \ldots \tau\left(g^{d-2}, g\right) \tau\left(g^{d-1}, g\right) \\
& \cdot e(i, i+(d-1) m) \cdot p \cdot e(i+(d-1) m, i) \cdot \overline{1} \\
= & p \cdot \tau(g, g) \tau\left(g^{2}, g\right) \ldots \tau\left(g^{d-2}, g\right) \tau\left(g^{d-1}, g\right) \\
= & \quad \cdot e(i, i) \cdot \overline{1} \cdot e(i, i) \overline{1} .
\end{aligned}
$$


It now follows that $\left\langle X^{d}-p v\right\rangle \subset \operatorname{ker}(\psi)$, and a rank argument shows that

$$
\frac{S[X]}{\left\langle X^{d}-p v\right\rangle} \cong e_{i} \Gamma e_{i},
$$

as desired.

Notation 5.5. Let $T=S[z]$, where $z^{d}=p v$. Let $L=T \cdot z=z \cdot T$.

Corollary 5.6. $L$ is the unique maximal ideal of $T$ and contains $T \cdot p$. Moreover, $T / L \cong S / P$.

Proof. Since $T$ has an $S$-basis consisting of $1, z, \ldots, z^{d-1}$, then $L=P \oplus S z \oplus \cdots \oplus$ $S z^{d-1}$. Consequently $T / L \cong S / P$, and so $L$ is maximal. Clearly, $L$ contains $T \cdot p$ since $z^{d}=p v$. Suppose $M$ is a maximal ideal of $T$; then $L / M$ is a field containing $S /(S \cap M)$. The latter must be a field, and so $M$ contains $p$. Then $L^{d} \subset M$. Since $M$ is prime, $L \subset M$, and so $L=M$.

Since $G$ acts faithfully on $\left\{e_{1}, \ldots, e_{n}\right\}$ (i.e., there are no fixed elements), each orbit contains the same number of elements, namely $d=|G|$. Consequently, there are $m=n / d$ orbits. Let $i_{1}, \ldots, i_{m}$ denote the smallest positive integer in each orbit, and set $\epsilon_{j}=\sum_{x \in G} e\left({ }^{x} i_{j},{ }^{x} i_{j}\right)$; that is, $\epsilon_{j}$ is the sum of all the $e_{i}$ for which the $i$ belong to the same orbit as $i_{j}$.

Corollary 5.7. For any $1 \leq j \leq d$,

$$
\epsilon_{j} \Gamma \epsilon_{j} \cong M_{d}(T)
$$

where $M_{d}(T)$ denotes the full $d \times d$ matrix ring over $T$.

Proof. For $1 \leq t \leq n$, we know that $t$ belongs to the orbit of $i_{j}$ if and only if $e_{t} \Gamma \cong e_{i_{j}} \Gamma$, by Lemma 5.3 Consequently, if $t$ belongs to the orbit of $i_{j}$, then $e_{t} \Gamma e_{t} \cong \operatorname{End}\left(e_{t} \Gamma\right) \cong \operatorname{End}\left(e_{i_{j}} \Gamma\right) \cong e_{i_{j}} \Gamma e_{i_{j}}$. By Lemma 5.4, $e_{i_{j}} \Gamma e_{i_{j}} \cong T$. Now

$$
\epsilon_{j} \Gamma=\bigoplus_{x \in G}^{x} e_{j} \Gamma
$$

which is a right $\Gamma$-module isomorphic to $d$ copies of $e_{j} \Gamma$. On the one hand the endomorphism ring is $\epsilon_{j} \Gamma \epsilon_{j}$ (acting by left multiplication); from general principles the endomorphism ring is isomorphic to the $d \times d$ matrix ring over the endomorphism $\operatorname{ring} T=e_{i_{j}} \Gamma e_{i_{j}}$.

Proposition 5.8. Let $i$ and $j$ be the smallest positive integers in their respective orbits $^{G} i$ and ${ }^{G} j$. Then:

1. If $i \leq j$, then $e_{i} \Gamma e_{j}=e_{i} \Gamma e_{i}(e(i, j) \cdot \overline{1})=(e(i, j) \cdot \overline{1}) e_{j} \Gamma e_{j}$, and this is isomorphic to $T$ as graded $e_{i} \Gamma e_{i}-e_{j} \Gamma e_{j}$-bimodules.

2. If $i>j$, then $e_{i} \Gamma e_{j}=e_{i} \gamma e_{i}(e(i, j+m) \cdot \bar{g})=(e(i, j+m) \cdot \bar{g}) e_{j} \Gamma e_{j}$, and this is isomorphic to $L=T z$ as graded $e_{i} \Gamma e_{i}-e_{j} \Gamma e_{j}$-bimodules.

Proof. We adopt the notation from the proof of Theorem 5.4. In particular, since $i$ is the smallest positive integer in ${ }^{G} i$ and since $\alpha(g)=\iota_{u^{m}}$, we have that

$$
\begin{aligned}
i \leq m & <{ }^{g} i=i+m \leq 2 m<{ }^{g^{2}} i=i+2 m \leq 3 m<\ldots \\
& <{ }^{g^{d-1}} i=i+(d-1) m \leq d m=n
\end{aligned}
$$


A similar chain of inequalities holds for $j$. Using an argument analogous to that found in the proof of Theorem 5.4 we have, for $0 \leq j \leq d-2$,

$$
\alpha\left(g^{k}\right)(e(i, j+m))=\iota_{u^{m k}}(e(i, j+m))=e(i+m k, i+m(k+1))
$$

while

$$
\begin{aligned}
\alpha\left(g^{d-1}\right)(e(i, j+m)) & =\iota_{u^{m(d-1)}}(e(i, j+m)) \\
& =p \cdot e(i+m(d-1), j+m(d)-n) \\
& =p \cdot e(i+m(d-1), j) .
\end{aligned}
$$

Next we find an $S$-basis for $e_{i} \Gamma e_{j}$. We have

$$
\begin{aligned}
e_{i} \Gamma e_{j} & =\left\{\sum_{x \in G} e_{i} \lambda_{x} \bar{x} e_{j} \mid \lambda_{x} \in \Lambda\right\} \\
& =\left\{\sum_{x \in G} e_{i} \lambda_{x} e_{x} \bar{x} \mid \lambda_{x} \in \Lambda\right\} \\
& =\left\{\sum_{x \in G} e\left(i,{ }^{x} j\right) s\left(i,{ }^{x} j, x\right) \bar{x}\right\} \\
& =\left\{\sum_{k=0}^{d-1} e(i, j+m k) s(i, j+m k, k) \overline{g^{k}}\right\},
\end{aligned}
$$

where $x=g^{k}$ and $s\left(i,{ }^{x} j, x\right)=s(i, j+m k, k)$ is the $\left(i,{ }^{x} j\right)$-entry of $l_{x}$.

(1) Assume that $i \leq j$. Then from $(*)$ we see that

$$
\begin{aligned}
i \leq j & <{ }^{g} i=i+m \leq{ }^{g} j=j+m<\ldots \\
& <{ }^{g^{d-1}} i=i+m(d-1) \leq{ }^{g^{d-1}} j=j+m(d-1)
\end{aligned}
$$

so that, in particular, $i<{ }^{x} j$ for all $x \in G$. Thus, by $(* *)$, we see that the elements $s\left(i,{ }^{x} j, x\right)=s(i, j+m k, k)$ can be ANY elements from $S$, and so a basis for $e_{i} \Gamma e_{j}$ is $e(i, j) \overline{1}, e(i, j+m) \bar{g}, e(i, j+m 2) \bar{g}^{2}, \ldots, e(i, j+m(d-1)) \bar{g}^{d-1}$.

Next we claim that $e_{i} \Gamma e_{j}=e_{i} \Gamma e_{i}(e(i, j) \overline{1})=(e(i, j) \overline{1}) e_{j} \Gamma e_{j}$. It is clear that

$$
e_{i} \Gamma e_{i}(e(i, j) \overline{1}),(e(i, j) \overline{1}) e_{j} \Gamma e_{j} \subset e_{i} \Gamma e_{j} .
$$

Given any basis element $e(i, j+m k) \bar{g}^{k}$ of $e_{i} \Gamma e_{j}$, where $0 \leq k \leq d-1$, we have

$$
\begin{aligned}
e(i, j+m k) \bar{g}^{k} & =e(i, i+m k) e(i+m k, j+m k) \bar{g}^{k} \\
& =e(i, i+m k) \alpha\left(g^{k}\right)(e(i, j)) \bar{g}^{k} \\
& =e(i, i+m k) \bar{g}^{k} e(i, j) \overline{1},
\end{aligned}
$$

which shows that $e_{i} \Gamma e_{j} \subset e_{i} \Gamma e_{i}(e(i, j) \overline{1})$. Similarly,

$$
e(i, j) \overline{1} e(j, j+m k) \bar{g}^{k}=e(i, j+m k) \bar{g}^{k},
$$

so that $e_{i} \Gamma e_{j} \subset(e(i, j) \overline{1}) e_{j} \Gamma e_{j}$.

Since $e_{i} \Gamma e_{j}=e_{i} \Gamma e_{i}(e(i, j) \overline{1})=(e(i, j) \overline{1}) e_{j} \Gamma e_{j}$ and $T \cong e_{i} \Gamma e_{i} \cong e_{j} \Gamma e_{j}$, it is possible to define a $T$-module map $\theta: e_{i} \Gamma e_{j} \rightarrow T$ by mapping $e(i, j) \overline{1}$ to the identity of $T$. This is a graded $\left(e_{i} \Gamma e_{i}\right)-\left(e_{j} \Gamma e_{j}\right)$-bimodule isomorphism.

(2) Assume that $i>j$. Again, since $i$ and $j$ are the smallest positive integers in their orbits, we see from $(*)$ that

$$
\begin{aligned}
j<i<{ }^{g} j & =j+m \leq{ }^{g} i=i+m<\ldots \\
& <{ }^{g^{d-1}} j=j+m(d-1) \leq{ }^{g^{d-1}} i=i+m(d-1)
\end{aligned}
$$


so that, in particular, $i<{ }^{x} j$ for all $x \in G \backslash\{1\}$. Thus, by $(* *)$, we see that the elements $s(i, j+m k, k)$ can be ANY elements from $S$, when $1 \leq k \leq d-1$, but must belong to $P$ when $k=0$ (since $i>j$ ). Thus, a basis for $e_{i} \Gamma e_{j}$ is $p \cdot e(i, j) \overline{1}, e(i, j+m) \bar{g}, e(i, j+2 m) \bar{g}^{2}, \ldots, e(i, j+(d-1) m) \bar{g}^{d-1}$.

Next we claim that $e_{i} \Gamma e_{j}=e_{i} \Gamma e_{i}(e(i, j+m) \bar{g})=(e(i, j+m) \bar{g}) e_{j} \Gamma e_{j}$. It is clear that $e_{i} \Gamma e_{i}(e(i, j+m) \bar{g}),(e(i, j+m) \bar{g}) e_{j} \Gamma e_{j} \subset e_{i} \Gamma e_{j}$. Given any basis element $e(i, j+m k) \bar{g}^{k}$ of $e_{i} \Gamma e_{j}$, where $1 \leq k \leq d-1$, we have

$$
\begin{aligned}
e(i, j+m k) \bar{g}^{k} & =e(i, i+m(k-1)) e(i+m(k-1), j+m k) \bar{g}^{k} \\
& =e(i, i+m(k-1)) \alpha\left(g^{k-1}\right)(e(i, j+m)) \bar{g}^{k} \\
& =\tau\left(g^{k-1}, g\right)^{-1} e(i, i+m(k-1)) \bar{g}^{k-1} e(i, j+m) \bar{g} \\
& =\left(e_{i} \tau\left(g^{k-1}, g\right)^{-1} \bar{g}^{k-1} e_{i}\right) \cdot e(i, j+m) \bar{g}
\end{aligned}
$$

This shows that the basis element $e(i, j+m k) \bar{g}^{k}$ of $e_{i} \Gamma e_{j}$, where $1 \leq k \leq d-1$, belongs to $e_{i} \Gamma e_{i}(e(i, j+m) \bar{g})$. The basis element $p e(i, j) \overline{1}$ also belongs to $e_{i} \Gamma e_{i}(e(i, j+m) \bar{g})$, because

$$
\begin{aligned}
p e(i, j) \overline{1} & =e(i, i+m(d-1)) p e(i+m(d-1), j) \overline{1} \\
& =e(i, i+m(d-1)) \alpha\left(g^{d-1}\right)(e(i, j+m)) \overline{1} \\
& =\tau\left(g^{d-1}, g\right)^{-1} e(i, i+m(d-1)) \bar{g}^{d-1} e(i, j+m) \bar{g} \\
& =\left(e_{i} \tau\left(g^{d-1}, g\right)^{-1} \bar{g}^{d-1} e_{i}\right) e(i, j+m) \bar{g} .
\end{aligned}
$$

This proves the claim that $e_{i} \Gamma e_{j}=e_{i} \Gamma e_{i}(e(i, j+m) \bar{g})$. A similar argument shows that $e_{i} \Gamma e_{j}=(e(i, j+m) \bar{g}) e_{j} \Gamma e_{j}$.

Since $e_{i} \Gamma e_{j}=e_{i} \Gamma e_{i}(e(i, j+m) \bar{g})=(e(i, j+m) \bar{g}) e_{j} \Gamma e_{j}$ and $T \cong e_{i} \Gamma e_{i} \cong e_{j} \Gamma e_{j}$, it follows that there is a graded $\left(e_{i} \Gamma e_{i}\right)-\left(e_{j} \Gamma e_{j}\right)$-bimodule isomorphism $\theta: e_{i} \Gamma e_{j} \rightarrow T z$ by mapping $e(i, j+m) \bar{g}$ to $z$.

Theorem 5.9. As rings,

$$
\Gamma \cong\left(\begin{array}{cccc}
M_{d}(T) & M_{d}(T) & \ldots & M_{d}(T) \\
M_{d}(L) & M_{d}(T) & \cdots & M_{d}(T) \\
\vdots & \vdots & \vdots & \vdots \\
M_{d}(L) & \cdots & M_{d}(L) & M_{d}(T)
\end{array}\right)
$$

In particular, $\Lambda$ is graded Morita equivalent to

$$
\left(\begin{array}{cccc}
T & T & \ldots & T \\
L & T & \ldots & T \\
\vdots & \vdots & \vdots & \vdots \\
L & \ldots & L & T
\end{array}\right)
$$

Proof. Write $\Gamma_{\Gamma} \cong\left(e_{i_{1}} \Gamma\right)^{d} \oplus \cdots \oplus\left(e_{i_{m}} \Gamma\right)^{d}$. Since $\Gamma \cong \operatorname{End}\left(\Gamma_{\Gamma}\right)$ and since $\operatorname{End}\left(\Gamma_{\Gamma}\right)$ is isomorphic to

$$
\left(\begin{array}{cccc}
\left(e_{i_{1}} \Gamma e_{i_{1}}\right)_{d \times d} & \left(e_{i_{1}} \Gamma e_{i_{2}}\right)_{d \times d} & \ldots & \left(e_{i_{1}} \Gamma e_{i_{d}}\right)_{d \times d} \\
\left(e_{i_{2}} \Gamma e_{i_{1}}\right)_{d \times d} & \left(e_{i_{2}} \Gamma e_{i_{2}}\right)_{d \times d} & \ldots & \left(e_{i_{2}} \Gamma e_{i_{d}}\right)_{d \times d} \\
\vdots & \vdots & \vdots & \vdots \\
\left(e_{i_{d}} \Gamma e_{i_{1}}\right)_{d \times d} & \ldots & \left(e_{i_{d}} \Gamma e_{i_{d-1}}\right)_{d \times d} & \left(e_{i_{m}} \Gamma e_{i_{m}}\right)_{d \times d}
\end{array}\right)
$$

as rings, we see that $\Gamma$ is ring isomorphic to the matrix ring of $(*)$. It is straightforward to see that these are graded ring isomorphisms. Finally, $\Gamma$ is graded Morita 
equivalent to the matrix ring of $(* *)$ because $\left(e_{i_{1}}+e_{i_{2}}+\cdots+e_{i_{m}}\right) \Gamma$ is a graded right $\Gamma$-progenerator whose endomorphism ring is $(* *)$.

We give a general result needed to show that $T$ is a maximal order.

Lemma 5.10. Let $R$ be a Dedekind ring with quotient field $K$, and $S$ the integral closure of $R$ in a separable field extension $L$ of $K$. Suppose there is an element $\theta \in S$ such that $L=K(\theta)$. Let $\Delta(\theta)$ be the discriminant of the basis $1, \theta, \cdots, \theta^{n-1}$, where $[L: K]=n$. Then

$$
\Delta(\theta) S \subseteq R[\theta] \subseteq S .
$$

Proof. This can be found in the proof of [14, Theorem 10.3, p. 127].

Theorem 5.11 (Eisenstein's Theorem). Let $R$ be a complete DVR with maximal ideal $\pi R$ and quotient field $K$. Let

$$
f(X)=X^{n}+a_{n-1} X^{n-1}+\cdots+a_{0}
$$

be an Eisenstein polynomial (so $a_{i} \in R, \pi \mid a_{i}$ for all $i$ and $\pi^{2} \backslash \chi_{0}$ ). Let $\theta$ be a root of $f(X)$, and $L=K(\theta)$, and $S$ the integral closure of $R$ in $L$. Then

1. $S=R[\theta]$;

2. $\theta S$ is the unique maximal ideal of $S$;

3. $\theta S$ has ramification index $e=n$ and residue class degree $f=1$.

Proof. This is a well-known result.

Corollary 5.12. The ring $T$ is a maximal order.

Proof. Let $f(X)=X^{d}-p v$ and observe that $f(X)$ satisfies Eisenstein's Theorem. Let $z$ be a root of $f(X)$, and set $F=K(z)$. Let $\tilde{T}$ denote the integral closure of $S$ inside $F$; this is also the integral closure of $T$ inside $F$. By Eisenstein's theorem (Theorem [5.11), $\tilde{T}=S[z]=T$.

We are now ready to prove Theorem D from the introduction.

Theorem 5.13. Let $S$ be a complete DVR with maximal ideal $p S$ and quotient field $K$. Let $\Lambda$ be a basic hereditary $S$-order inside $M_{n}(K)$. If $G$ is a group that acts on $\Lambda$ as central outer automorphisms via the action $\alpha: G \rightarrow \operatorname{Aut}(\Lambda)$ and factor set $\tau \in Z^{2}\left(G, S^{\bullet}\right)$, then the crossed product $\Lambda *_{\tau}^{\alpha} G$ is hereditary and has finite representation type.

Proof. By the structure theorem for hereditary orders ([14, Theorem 39.14]) and the fact that $T$ is a maximal order with maximal ideal $L$, then $\Lambda *_{\tau}^{\alpha} G$ is hereditary from Theorem 5.9. Since hereditary orders have finite representation type, the proof is complete.

We return a final time to the quaternion example.

Example 5.14. Assume the notation of Examples 3.3, 3.7 and 4.5 We claim that

$$
\Gamma=(S \otimes \Delta) * G \cong M_{2}(T)
$$

where $T=S[X] /\left\langle X^{2}-2 v\right\rangle$. The reason for this is that Outcent $(S \otimes \Delta) \cong \mathbf{Z} / 2 \mathbf{Z}$, and so $\alpha: G \rightarrow \operatorname{Autcent}(S \otimes \Delta)$ extends to an isomorphism $\hat{\alpha}: G \rightarrow \operatorname{Outcent}(S \otimes \Delta)$. Hence $G=\langle g\rangle$ is cyclic of order 2. The element $v$ is the unit of $R$ such that $\bar{g}^{2}=v$. Consequently, $n=2$ and $d=2$. The claim now follows from Theorem 5.9] Thus, in this example, $(S \otimes \Delta) * G$ is maximal. 
We remark that the above example provides a counterexample to the only if statement from [8] Theorem 4.2], which asserts that a $G$-strongly graded order $\Lambda$ is maximal if and only if $\Lambda_{1}$ is maximal and contains the order of the group as a unit. As we have seen in Theorem [2.4 this theorem is true when the group is acting as inner automorphisms.

Finally, we can prove Theorem $\mathrm{C}$ from the introduction.

Theorem 5.15. Let $R$ be a complete local Dedekind domain with quotient field $K$ which is a local field. Let $\Delta$ be a maximal $R$-order inside a central $K$-division ring $D$. If $G$ is a group that acts on $\Delta$ as central outer automorphisms via the action $\alpha: G \rightarrow \operatorname{Aut}(\Delta)$ and factor set $\tau \in Z^{2}\left(G, R^{\bullet}\right)$, then the crossed product order $\Delta *_{\tau}^{\alpha} G$ is hereditary and has finite representation type.

Proof. We can apply Corollary 4.15 to conclude that $\Delta *_{\tau}^{\alpha} G$ is hereditary if and only if $\Lambda *_{\tau^{\prime}}^{\alpha^{\prime}} G$ is hereditary, where $\alpha^{\prime}=\Phi(1 \otimes \alpha(-)) \Phi^{-1}$ is an outer action and $\tau^{\prime}=\tau$ is a factor set. But $\Lambda *_{\tau^{\prime}}^{\alpha^{\prime}} G$ is hereditary from Theorem [5.13.

\section{The global and Central Simple CASES}

In this final section, we prove our main result, Theorem A from the introduction. Having shown that outer actions on maximal orders inside division rings give rise to hereditary crossed product orders, we extend this result to outer actions on maximal orders inside central simple $K$-algebras. We assume that Notation 3.1 holds throughout this section. In particular, $\Gamma=M_{q}(\Delta)$, where $\Delta$ denotes a maximal order inside a division ring $D$. Our first line of argument is to show that Outcent $(\Gamma)$ is isomorphic to $\operatorname{Outcent}(\Delta)$. To do this, we need to introduce some additional notation.

Notation 6.1. Let $T$ and $T^{\prime}$ denote unital rings. If $f: T \rightarrow T^{\prime}$ is a ring homomorphism, then define $\chi(f): M_{q}(T) \rightarrow M_{q}\left(T^{\prime}\right)$ via $\chi(f)(X)=\chi(f)\left(x_{i, j}\right)=\left(f\left(x_{i, j}\right)\right)$, where $X=\left(x_{i, j}\right)$. In the case where $f \in \operatorname{Aut}(T)$, this gives rise to a group monomorphism $\chi: \operatorname{Aut}(T) \rightarrow \operatorname{Aut}\left(M_{q}(T)\right)$.

Define $F: S \otimes M_{q}(\Delta) \rightarrow M_{q}(S \otimes \Delta)$ via $F\left(s \otimes\left(\delta_{i, j}\right)\right)=\left(s \otimes \delta_{i, j}\right)$, which is a ring isomorphism. Since $\chi(\Phi): M_{q}(S \otimes \Delta) \rightarrow M_{q}(\Lambda)$ is a ring isomorphism, we have the ring isomorphism $\chi(\Phi) F: S \otimes M_{q}(\Delta) \rightarrow M_{q}(\Lambda)$.

It follows that we have a group homomorphism $F(1 \otimes-) F^{-1}: \operatorname{Aut}(\Gamma) \rightarrow$ $\operatorname{Aut}\left(M_{q}(S \otimes \Delta)\right)$ via $F(1 \otimes \sigma) F^{-1}((s \otimes \delta) e(i, j))=s \otimes \sigma(\delta e(i, j))$, where $e(i, j)$ denotes the matrix with 1 in the $i, j$-entry and zeros elsewhere.

Proposition 6.2. The following diagram is commutative:

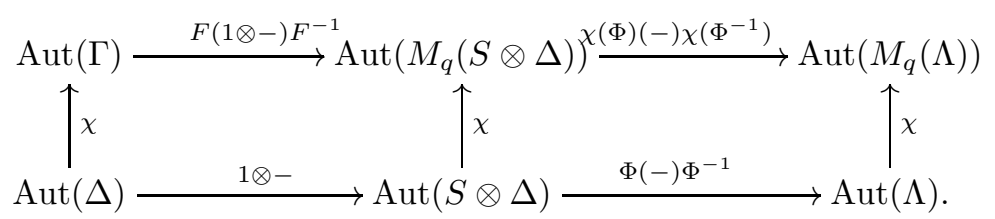

Proof. Since the right square is clearly commutative, it suffices to show that the left square commutes. To that end, let $\sigma \in \operatorname{Aut}(\Delta)$, and set $f=F(1 \otimes \chi(\sigma)) F^{-1}$ and $g=\chi(1 \otimes \sigma)$. Let $X \in M_{q}(S \otimes \Delta)$ and write $X=\left(x_{i, j}\right)$, where $x_{i, j}=$ 
$\sum_{k} s_{i, j, k} \otimes \delta_{i, j, k}, s_{i, j, k} \in S$ and $\delta_{i, j, k} \in \Delta$. Then

$$
\begin{aligned}
f(X) & =\left(F(1 \otimes \chi(\sigma)) F^{-1}\right)\left(\sum_{i, j, k}\left(s_{i, j, k} \otimes \delta_{i, j, k}\right) e_{i, j}\right) \\
& =\sum_{i, j, k} s_{i, j, k} \otimes \chi(\sigma)\left(\delta_{i, j, k} e_{i, j}\right) \\
& =\sum_{i, j, k} s_{i, j, k} \otimes \sigma\left(\delta_{i, j, k}\right) e_{i, j} \\
& =\sum_{i, j, k}(1 \otimes \sigma)\left(s_{i, j, k} \otimes \delta_{i, j, k}\right) e_{i, j} \\
& =\sum_{i, j, k} \chi(1 \otimes \sigma)\left(s_{i, j, k} \otimes \delta_{i, j, k} e_{i, j}\right) \\
& =\chi(1 \otimes \sigma)\left(\sum_{i, j, k} s_{i, j, k} \otimes \delta_{i, j, k} e_{i, j}\right) \\
& =g(X),
\end{aligned}
$$

which proves commutativity.

If $\Omega$ denotes an $R$-order inside a central $K$-algebra, then there is a natural group isomorphism $P \otimes_{M_{q}(\Omega)}-\otimes_{M_{q}(\Omega)} P^{-1}: \operatorname{Pic}\left(M_{q}(\Omega)\right) \rightarrow \operatorname{Pic}(\Omega)$, where $P=e M_{q}(\Omega)$, $P^{-1}=M_{q}(\Omega) e$ and $e=e(1,1)$. (Actually, this holds for any Morita equivalent rings.) Recall, from Definition 4.11, the group monomorphism $\Psi_{\Omega}$ : Outcent $(\Omega) \rightarrow$ $\operatorname{Picent}(\Omega)$, where $\Psi_{\Omega}(\sigma)=\left[\Omega_{\sigma}\right]$.

Proposition 6.3. Let $\Omega$ denote an $R$-order inside a central $K$-algebra such that $\Psi_{\Omega}$ is an isomorphism. There is a commutative diagram of group isomorphisms

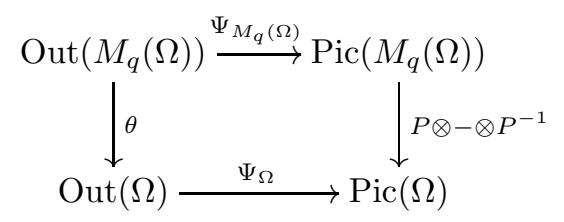

where $\theta=\left(\Psi_{\Omega}\right)^{-1}\left(P \otimes-\otimes P^{-1}\right)\left(\Psi_{M_{q}(\Omega)}\right)$ and $\theta^{-1}=\chi$. In particular, $\theta$, $\chi$, and $\Psi_{M_{q}(\Omega)}$ are isomorphisms.

Proof. Let $T=M_{q}(\Omega)$. Observe that $\theta$ is monic. We claim that $\chi$ is monic. Suppose that $1 \neq \operatorname{Inn}(\Omega) \sigma \in \operatorname{Out}(\Omega)$ but $\chi(\sigma)$ is inner. This means that there is a $C \in T^{\bullet}$ such that $\chi(\sigma)=\iota_{C}$. By Lemma 4.2, there exists $u \in(K \Omega)^{\bullet}$ such that $\sigma=\iota_{u}$. Thus, $\chi(\sigma)=\chi\left(\iota_{u}\right)=\iota_{u I}=\iota_{C}$. It follows that $\iota_{C}^{-1} u I$ is the identity automorphism and so $C^{-1} u I$ belongs to the center of $K \cdot T$, which is $K$. Thus, $\mu u I=C$ for some $\mu \in K^{\bullet}$, and so $\mu u I \in M_{q}(\Omega)^{\bullet}$. This implies $\omega=\mu u \in \Omega^{\bullet}$. Since $\mu$ is central, $\iota_{u}=\iota_{\mu u}$, and thus $\sigma=\iota_{\mu u} \in \operatorname{Inn}(\Omega)$. This is a contradiction, so $\chi$ is monic.

Next we show that $\theta \chi=1$. Let $\sigma \in \operatorname{Aut}(\Omega)$. Then $\theta \chi(\operatorname{Inn}(\Omega) \sigma)=\operatorname{Inn}(\Omega) \beta$, so that $\Omega_{\beta} \cong e T_{\chi(\sigma)} e$ as $\Omega$-bimodules. But $\chi(\sigma)$ fixes $e$ since $\sigma$ fixes 1 , and so $e T_{\chi(\sigma)} e \cong e T e_{\sigma} \cong \Omega_{\sigma}$. Hence $\operatorname{Inn}(\Omega) \sigma=\operatorname{Inn}(\Omega) \beta$, which completes the proof. Consequently $\theta$ is an isomorphism, and so $\chi=\theta^{-1}$. 
Corollary 6.4. There exists a commutative diagram

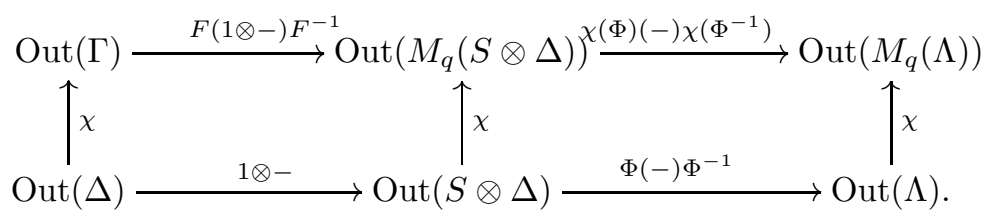

where each map is an isomorphism.

Proof. By the above Lemma 6.3 each vertical map $\chi$ is an isomorphism. By Theorem 4.2, each map in the bottom row is an isomorphism, and so by the commutativity of the diagram (from Proposition 6.2), the top row is also an isomorphism.

From Proposition 4.10, we know that $\operatorname{Autcent}(\Lambda)$ is the semidirect product of $\operatorname{Inn}(\Lambda)$ and $\mathcal{O}$. It is not surprising that a similar result holds for Autcent $\left(M_{q}(\Lambda)\right)$.

Corollary 6.5. Autcent $\left(M_{q}(\Lambda)\right)$ is the semidirect product of $\operatorname{Inn}\left(M_{q}(\Lambda)\right)$ and $\chi(\mathcal{O})$.

Proof. We know that $\chi: \operatorname{Autcent}(\Lambda) \rightarrow \operatorname{Autcent}\left(M_{q}(\Lambda)\right)$ is a monomorphism which induces an isomorphism $\chi$ : Outcent $(\Lambda) \rightarrow \operatorname{Outcent}\left(M_{q}(\Lambda)\right)$ by Corollary 6.4 Since Autcent $(\Lambda)$ is a semidirect product of $\operatorname{Inn}(\Lambda)$ by $\mathcal{O}$, the result follows.

We next present our first reduction technique, which will allow us to pass the hereditary problem from $M_{q}(\Lambda) *_{\tau}^{\alpha} G$ to $\Lambda *_{\tau}^{\alpha^{\prime}} G$.

Theorem 6.6. Each outer action $\alpha: G \rightarrow \operatorname{Autcent}\left(M_{q}(\Lambda)\right)$ of $G$ on $\Omega=M_{q}(\Lambda)$ with factor set $\tau \in Z^{2}\left(G, S^{\bullet}\right)$ induces an outer action of $G$ on $\Lambda$ via $\alpha^{\prime}: G \rightarrow$ Aut $(\Lambda)$ with factor set $\tau^{\prime} \in Z^{2}\left(G, S^{\bullet}\right)$, and conversely. Moreover, the crossed products $\Omega *{ }_{\tau}^{\alpha} G$ and $\Lambda *_{\tau^{\prime}}^{\alpha^{\prime}} G$ made with the corresponding outer actions and twistings are Morita equivalent.

Proof. $(\Rightarrow)$ Set $\Omega(G)=\Omega *_{\tau}^{\alpha} G$. By Corollary 6.5 and Theorem[5.1, we can assume that $\alpha: G \rightarrow \chi(\mathcal{O})$. Define $\alpha^{\prime}=\chi^{-1} \alpha: G \rightarrow \mathcal{O} \subset \operatorname{Autcent}(\Lambda)$. Since $\alpha^{\prime}$ is a group monomorphism, $\alpha^{\prime}$ defines an outer action of $G$ on $\Lambda$. We can write $\alpha(g)=\chi\left(\alpha^{\prime}(g)\right)$. In particular, observe that, for any standard matrix idempotent $e_{i}=e(i, i)$ in $\Omega$, we have

$$
\alpha(g)\left(e_{i}\right)=\chi\left(\alpha^{\prime}(g)\right)\left(e_{i}\right)=\alpha^{\prime}(g)(1) e_{i}=e_{i},
$$

because the automorphism $\alpha^{\prime}(g)$ fixes the identity 1 of $\Lambda$.

As usual, let $\bar{g}$ denote a basis element for $\Omega * G$. As a result of $(*)$, each $\bar{g}$ commutes with $e_{i}$, because

$$
\bar{g} e_{i}=\alpha(g)\left(e_{i}\right) \bar{g}=e_{i} \bar{g} .
$$

Let $e=e(1,1)$, the matrix idempotent inside $\Omega=M_{q}(\Lambda)$ with 1 in the $(1,1)$ entry and zeros elsewhere. We will identify $\Lambda$ with $e \Omega e$ via the map $\lambda \mapsto \lambda e=e \lambda$. We can construct $\Lambda *_{\tau}^{\alpha^{\prime}} G$, which we denote by $\Lambda(G)$. The basis elements for $\Omega^{\prime}$ are of the form $e \bar{g}$, where $g \in G$. By our observation $(* *),\left(e \bar{g}^{-1}\right)=(e \bar{g})^{-1}$ for all $g \in G$. 
We claim that $(e \bar{g}) \lambda\left(e \bar{g}^{-1}\right)=\alpha^{\prime}(\lambda) e$ for all $\lambda \in \Lambda$. But using $(* *)$, we have

$$
\begin{aligned}
(e \bar{g}) \lambda\left(e \bar{g}^{-1}\right) & =e\left(\bar{g} \lambda \bar{g}^{-1}\right) e \\
& =e \alpha(\lambda) e \\
& =e \chi\left(\alpha^{\prime}\right)(\lambda) e \\
& =\alpha^{\prime}(\lambda) e
\end{aligned}
$$

Next we claim that $(e \bar{g})(e \bar{h})=\alpha(g, h) e \overline{g h}$. But

$$
(e \bar{g})(e \bar{h})=e \bar{g} \bar{h}=e \alpha(g, h) \overline{(} g h)=\alpha(g, h) e \overline{g h} .
$$

Finally, we show that $\Omega *_{\tau}^{\alpha} G$ and $\Lambda *_{\tau}^{\alpha^{\prime}} G$ are Morita equivalent. In fact, we prove a stronger condition, namely, that they are graded equivalent; see [8] for details about graded equivalence. Set $P=\Lambda^{(q)}$, which we make into a $(\Lambda, \Omega)$ bimodule in the usual way, and set $\tilde{P}=P \otimes_{\Omega} \Omega(G)$. We have a grading on $\tilde{P}$ via $\tilde{P}=\bigoplus_{x \in G} P \otimes \bar{x}$.

We claim that $\tilde{P}$ is a $(\Lambda(G), \Omega(G))$-bimodule. It suffices to show that $\tilde{P}$ is a left $\Lambda(G)$-module. But, for $x \in G$, first define an automorphism of $P$ induced from $\alpha^{\prime}(x)$, which we denote by $\chi \alpha^{\prime}(x)$. That is, for $p=\left(\lambda_{1}, \ldots, \lambda_{q}\right)$, set $\alpha^{\prime}(x)(p)=$ $\left(\alpha^{\prime}(x)\left(\lambda_{1}\right), \ldots, \alpha^{\prime}(x)\left(\lambda_{n}\right)\right)$. (Note that this is another induced automorphism from $\alpha^{\prime}(g)$.) Now define the $\Lambda(G)$-action on $\tilde{P}$ via

$$
(e \bar{x}) \cdot(p \otimes \bar{g})=\chi\left(\alpha^{\prime}(x)\right)(p) \otimes \overline{x g}=\chi\left(\alpha^{\prime}(x)\right)(p) \otimes \tau(x, g) \overline{x g} .
$$

We leave it to the reader to verify that associativity holds.

It follows that $\tilde{P}$ is a $G$-graded $(\Lambda(G), \Omega(G))$-bimodule, where $\tilde{P}_{g}=P \otimes \bar{g}$ and $\Lambda(e \bar{x}) \cdot \tilde{P}_{g} \cdot \Omega \bar{y}=\tilde{P}_{x g y}$.

Next we claim that ${ }_{\Lambda(G)} \tilde{P} \cong{ }_{\Lambda(G)}(\Lambda(G))^{(q)}$. Observe that $\Omega(G) \subset \operatorname{End}\left({ }_{\Lambda(G)} \tilde{P}\right)$ via the ring monomorphism $z \mapsto \rho_{z}$, where $\rho_{z}$ denotes right multiplication by $z \in$ $\Omega(G)$. Now since $e_{1}+\cdots+e_{q}$ is the identity matrix in $\Omega$, we have that $\tilde{P}=\bigoplus_{i=1}^{q} \tilde{P} e_{i}$ as left $\Lambda(G)$-modules. Now each $\tilde{P} e_{i}=\bigoplus_{g \in G} P \otimes \bar{g} e_{i}=\bigoplus_{g \in G}(\Lambda)^{(q)} \otimes e_{i} \bar{g} \cong$ $\bigoplus_{g \in G} \Lambda \otimes \bar{g} \cong \Lambda(G)$. Consequently, $\tilde{P}=\bigoplus_{i=1}^{q} \tilde{P} e_{i} \cong(\Lambda(G))^{(q)}$.

Thus, $\tilde{P}$ is a left, graded $\Lambda(G)$-module that generates $\Lambda(G)$-mod and is such that ${ }_{\Lambda(G)} \tilde{P}$ is projective. Hence, in the notation of $[8$, Theorem 2.6], $\tilde{P}$ is a graded, locally projective generator, and so $\Lambda(G)$ and $\operatorname{End}\left(\Lambda_{(G)} \tilde{P}\right)$ are graded equivalent. In particular, these two rings are Morita equivalent.

Finally, we show that $\Omega(G) \cong \operatorname{End}\left({ }_{\Lambda(G)} \tilde{P}\right)$ via the ring homomorphism $\rho: z \mapsto$ $\rho_{z}$. To see that $\rho$ is injective, suppose $\tilde{p} z=0$ for all $\tilde{p} \in \tilde{P}$ and for some $z \in \Omega(G)$. But then $e(1, i) z=0$ for all $i=1, \ldots, q$, and so $z=0$. For surjectivity, we identify $\operatorname{End}\left({ }_{\Lambda(G)} \tilde{P}\right)$ with $M_{q}(\Lambda(G))$, since ${ }_{\Lambda(G)} \tilde{P} \cong{ }_{\Lambda(G)}(\Lambda(G))^{(q)}$. Suppose $\beta \in M_{q}(\Lambda(G))$, and write $\beta=\left(\beta_{i, j}\right)$, where $\beta_{i, j}=\sum_{g \in G} \beta_{i, j, g} e \bar{g}$. Define $T: M_{q}(\Lambda(G)) \rightarrow \Omega(G)$ via $\beta \mapsto \sum_{g \in G}\left(\beta_{i, j, g}\right) \bar{g}$, where we view $\left(\beta_{i, j, g}\right) \in \Omega$. We show that $T$ is the inverse of $\rho$. Let $z \in \Omega(G)$ and write $z=\sum_{g \in G} z_{g} \bar{g}$, where $z_{g}=\left(z_{g, i, j}\right) \in \Omega$. Then $\rho_{z}=\left(\left(\rho_{z}\right)_{i, j}\right)$, where $\left(\rho_{z}\right)_{i, j}: \tilde{P} e(1, i) \rightarrow \tilde{P} e(1, j)$ for all $i, j$ via $\left(\rho_{z}\right)_{i, j}=\rho_{e_{i} z e_{j}}$. But $e_{i} z e_{j}=e_{i} \sum_{g \in G} z_{g} \bar{g} e_{j}=\sum_{g \in G} e_{i} z_{g} e_{j} \bar{g}$ by $(* *)$. Hence,

$$
z \stackrel{\rho}{\longrightarrow} \rho_{z}=\sum_{g \in G} e_{i} z e_{j} \bar{g}=\sum_{g \in G} e_{i} z_{g} e_{j} \bar{g}=z,
$$


which shows that $T \rho$ is the identity map on $\Omega(G)$. In a similar way, $\rho T$ is the identity map on $M_{q}\left(\Lambda(G)\right.$ ), and so we have a ring isomorphism $\Omega(G) \cong M_{q}(\Lambda(G)$ ). This completes the proof that $\Omega(G)$ and $\Lambda(G)$ are (graded) Morita equivalent.

$(\Leftarrow)$ Suppose $G$ acts as outer automorphisms on $\Lambda$; that is, there is a group homomorphism $\alpha^{\prime}: G \rightarrow \operatorname{Aut}(\Lambda)$ such that $\alpha^{\prime}(G) \cap \operatorname{Inn}(\Lambda)=1$. Define $\tilde{\alpha}^{\prime}: G \rightarrow$ Autcent $\left(M_{q}(\Lambda)\right)$ via $\tilde{\alpha}^{\prime}=\chi(\alpha)$. If $\tilde{\alpha}^{\prime}(g) \in \operatorname{Inn}\left(M_{q}(\Lambda)\right)$, then $\alpha^{\prime}(g) \in \operatorname{Inn}(\Lambda)$ by Corollary 6.4, and so $g=1$. Hence, $G$ acts as outer automorphisms on $\Gamma$ by $\tilde{\alpha}^{\prime}$, as desired. Set $\tilde{\tau}=\tau$.

Next we show that $M_{q}(\Lambda) * \tilde{\tilde{\tau}}_{\tilde{\alpha^{\prime}}}^{\tilde{\alpha}^{\prime}} G$ is Morita equivalent to $\Lambda *_{\tau^{\prime}}^{\alpha^{\prime}} G$. To do this, we show that $M_{q}\left(\Lambda *_{\tau^{\prime}}^{\alpha^{\prime}} G\right) \cong M_{q}(\Lambda) *_{\tilde{\tau}}^{\tilde{\alpha}^{\prime}} G$. Toward that end, define $\eta: M_{q}\left(\Lambda *_{\tau^{\prime}}^{\alpha^{\prime}} G\right) \rightarrow$ $M_{q}(\Lambda) * \underset{\tilde{\tau}}{\tilde{\alpha}^{\prime}} G$ via

$$
\left(\gamma_{i, j}\right)=\left(\sum_{g \in G} \lambda_{i, j, g} \bar{g}\right) \mapsto \sum_{g \in G}\left(\lambda_{i, j, g}\right) \overline{\bar{g}}
$$

We leave it to the reader to verify that $\eta$ is a ring isomorphism.

Our second (and last) reduction enables us to pass the hereditary problem from $\Gamma *_{\tau}^{\alpha} G$ to $M_{q}(\Lambda) *_{\tau}^{\alpha^{\prime}} G$. When combined with the previous reduction technique, we can pass from $\Gamma *_{\tau}^{\alpha} G$ to $\Lambda * G$.

Theorem 6.7. Let $G$ be a group, let $\alpha: G \rightarrow \operatorname{Autcent}(\Gamma)$ denote a group homomorphism, and let $\tau: G \times G \rightarrow R^{\bullet}$ denote a factor set. Then:

1. $G$ acts as outer automorphisms on $\Gamma$ by $\alpha: G \rightarrow \operatorname{Autcent}(\Gamma)$ if and only if $G$ acts as outer automorphisms on $M_{q}(\Lambda)$ by the action

$$
\alpha^{\prime}=\chi(\Phi)\left(F(1 \otimes \alpha) F^{-1}\right) \chi\left(\Phi^{-1}\right): G \rightarrow \operatorname{Autcent}\left(M_{q}(\Lambda)\right) .
$$

2. $\Gamma *_{\tau}^{\alpha} G$ is hereditary if and only if $M_{q}(\Lambda) *_{\tau}^{\alpha^{\prime}} G$ is hereditary.

3. If $\alpha: G \rightarrow \operatorname{Autcent}(\Gamma)$ is an outer action of $G$ on $\Gamma$ and $\tau \in Z^{2}\left(G, R^{\bullet}\right)$ is a factor set, then there exist an outer action $\bar{\alpha}: G \rightarrow \operatorname{Autcent}(\Lambda)$ and a factor set $\bar{\tau} \in Z^{2}\left(G, S^{\bullet}\right)$ such that $\Gamma *_{\tau}^{\alpha} G$ is hereditary if and only if $\Lambda * \frac{\bar{\alpha}}{\tau} G$ is hereditary.

Proof. (1) Suppose $\alpha: G \rightarrow \operatorname{Aut}(\Gamma)$ is a group homomorphism such that $\alpha(G) \cap$ $\operatorname{Inn}(\Gamma)=1$. If $g \in G \backslash\{1\}$, then, since the top row of the diagram in Corollary 6.4 consists of isomorphisms, it follows that $\chi(\Phi)\left(F(1 \otimes \alpha(g)) F^{-1}\right) \chi\left(\Phi^{-1}\right) \notin$ $\operatorname{Inn}\left(M_{q}(\Lambda)\right)$. Consequently, the group homomorphism

$$
\alpha^{\prime}=\chi(\Phi)\left(F(1 \otimes \alpha) F^{-1}\right) \chi\left(\Phi^{-1}\right): G \rightarrow \operatorname{Aut}\left(M_{q}(\Lambda)\right)
$$

is an outer group action. The converse also holds in the same way.

(2) For $g \in G$, we have a commutative diagram of ring isomorphisms:

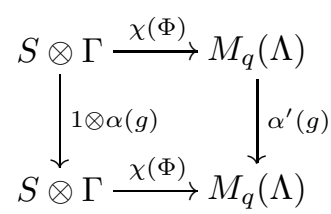

Thus, there is a ring isomorphism

$$
S \otimes\left(\Gamma *_{\tau}^{\alpha} G\right) \rightarrow M_{q}(\Lambda) *_{\tau^{\prime}}^{\alpha^{\prime}} G \text { via }\left(s \otimes\left(\delta_{i, j}\right)\right) \bar{g} \mapsto\left(s \otimes \delta_{i, j}\right) \overline{\bar{g}} .
$$

Now, applying Theorem [3.5 we see that $\Gamma *_{\tau}^{\alpha} G$ is hereditary if and only if $M_{q}(\Lambda) *_{\tau^{\prime}}^{\alpha^{\prime}} G$ is hereditary, as desired. 
(3) This follows from (1) and (2) above as well as Theorem 6.6.

We arrive at the proof of our global result, Theorem A from the introduction.

Theorem 6.8. Let $R$ denote a Dedekind domain whose quotient field $K$ is a global field. Let $\Gamma$ be an $R$-order inside a central simple $K$-algebra $A$, let $\alpha: G \rightarrow \operatorname{Aut}_{R}(\Gamma)$ be a group homomorphism, and let $\tau \in Z^{2}(G, R \bullet)$. Set $\Lambda=\Gamma *_{\tau}^{\alpha} G$. Then $\Lambda$ is hereditary if and only if, for each maximal ideal $P$ that contains a prime integer $p$ dividing $|G|$, any $p$-Sylow subgroup of $G$ acts as central outer automorphisms of $\hat{\Gamma}_{P}$ (i.e., the P-adic completion of $\Gamma$ ).

Proof. By [14, Theorem 40.5], $\Lambda$ is hereditary if and only if $\hat{\Lambda}_{P}$ is hereditary for all maximal ideals $P$ of $R$. We claim that $\Lambda$ is hereditary if and only if $\hat{\Lambda}_{P}$ is hereditary for those maximal ideals of $R$ that contain a prime integer $p$ that divides $|G|$. Fix a maximal ideal $P$ of $R$. If $|G|$ is relatively prime to $P$ (i.e., $P$ contains no prime integers that divide $|G|$ ), then $|G|$ is a unit in $\hat{R}_{P}$. Consequently, $\hat{\Lambda}_{P}$ is a separable extension of $\hat{\Gamma}_{P}$ by Proposition 2.2 (i.e., let $H=1$ ), and so, by Proposition 2.3, $\hat{\Lambda}_{P}$ is hereditary if and only if $\hat{\Gamma}_{P}$ is hereditary. But $\hat{\Gamma}_{P}$ is maximal, so $\hat{\Lambda}_{P}$ is hereditary in this case. This shows that $\Lambda$ is hereditary if and only if $\hat{\Lambda}_{P}$ is hereditary for those maximal ideals of $R$ that contain a prime integer $p$ that divides $|G|$.

Now suppose $P$ contains a prime integer $p$ that divides $|G|$, and assume $R$ is completed at $P$.

$(\Rightarrow)$ Assume that $\Lambda$ is hereditary. Let $Q$ denote a $p$-Sylow subgroup of $G$. Let $H$ denote the subgroup $\left(\left.\alpha\right|_{Q}\right)^{-1}(\operatorname{Inn}(\Gamma))$. By Proposition [2.3] $\Gamma * H$ is hereditary. Since $H$ acts on $\Gamma$ as inner automorphisms, we have, by Theorem 2.4 that $|H|$ is a unit in $R$. But $H$ is a $p$-group and $p$ is a non-unit in $R$, so we must have $|H|=1$. Consequently, $\left.\alpha\right|_{Q}: Q \rightarrow \operatorname{Aut}_{R}(\Gamma)$ is a monomorphism such that $\alpha(Q) \cap \operatorname{Inn}(\Gamma)=1$. This shows that $Q$ acts as central outer automorphisms of $\Gamma$.

$(\Leftarrow)$ Now assume that $Q$ acts as central outer automorphisms of $\Gamma$ for each $p$ Sylow subgroup of $G$. Since $|G: Q|$ is prime to $p,|G: H|$ is a unit of $R$ and so $\Gamma * G$ is a separable extension of $\Gamma * Q$, by Proposition 2.2 Hence, $\Gamma * G$ is hereditary if and only if $\Gamma * Q$ is hereditary, by Proposition [2.3. Now use Notation 3.1 to write $\Gamma=M_{q}(\Delta)$, where $\Delta$ is a maximal order inside a central $K$-skew field. By Theorem 6.7, $\Gamma * Q$ is hereditary if and only if $\Lambda * Q$ is hereditary where the action is outer and $\Lambda$ is as in Notation 4.6. Applying Theorem 5.13 gives us that $\Lambda * Q$ is hereditary, so the proof is complete.

\section{REFERENCES}

[1] G. Abrams and J. Haefner, Picard groups and infinite matrix rings, Trans. Amer. Math. Soc., 350 (1998), 2737-2752. MR 98j:16017

[2] L. Le Bruyn, M. Van den Bergh and F. Van Oystaeyen, Graded orders, Birkhäuser, Boston, 1988. MR 90h:16004]

[3] S. Chase, D.K. Harrison and A. Rosenberg, Galois theory and Galois cohomology of commutative rings, Mem. Amer. Math. Soc., 52 (1965), 15-33. MR 33:4118

[4] C. Curtis and I. Reiner, Methods of representation theory with applications to finite groups and orders, volume 1, John Wiley \& Sons, New York, 1981. MR 82i:20001

[5] C. Curtis and I. Reiner, Methods of representation theory with applications to finite groups and orders, volume 2, John Wiley \& Sons, New York, 1988. MR 88f:22002

[6] J. Haefner, Graded equivalence with applications, J. Alg. 172 (1995), 385-424. MR 96f:16052

[7] J. Haefner, Graded Morita theory for infinite groups, J. Alg., 169 (1994), 552-586. MR 95j:16051 
[8] J. Haefner, Reduction techniques for strongly graded rings and finite representation type, I: Bounded functors and graded equivalence, J. of Algebra 194 (1997), 567-593. MR 99a:16038

[9] G.J. Janusz, Tensor products of orders, J. London Math. Soc. 20 (1979), 186-192. MR 81g:16009

[10] S. Jøndrup, The group of automorphisms of certain subalgebras of matrix algebras, J. Alg., 141 (1991), 106-114. MR 92h:16034

[11] C. Năstăsescu, M. Van den Bergh, and F. Van Oystaeyen, Separable functors applied to graded rings, J. Alg. 123 (1989), 397-413. MR 90j:16001

[12] D. Passman, Infinite crossed products, Academic Press, Inc., New York, 1989. MR 90g:16002

[13] M.D. Rafael, Separable functors revisited, Comm. Alg. 18 (1990), 1445-1459. MR 91g:18001

[14] I. Reiner, Maximal orders, Academic Press, New York, 1975. MR 52:13901

Department of Mathematics, University of Colorado, Colorado Springs, Colorado 80933

E-mail address: haefner@math.uccs.edu

Department of Mathematics, University of Illinois, Champaign-Urbana, Illinois 61801

E-mail address: janusz@math.uiuc.edu 